\title{
Global Existence and Blow-up of Solutions for a System of Fractional Wave Equations
}

\begin{abstract}
Bashir Ahmad*, Ahmed Alsaedi, Mohamed Berbiche and Mokhtar Kirane
Abstract. We investigate the Cauchy problem for a $2 \times 2$-system of weakly coupled semi-linear fractional wave equations with polynomial nonlinearities posed in $\mathbb{R}^{+} \times \mathbb{R}^{N}$. Under appropriate conditions on the exponents and the fractional orders of the time derivatives, it is shown that there exists a threshold value of the dimension $N$, for which, small data-global solutions as well as finite time blowing-up solutions exist. Furthermore, we investigate the $L^{\infty}$-decay estimates of global solutions.
\end{abstract}

\section{Introduction}

We consider the following Cauchy problem

$$
\begin{cases}{ }^{C} D_{0 \mid t}^{\gamma_{1}} u-\Delta u=f(v(t, \cdot)), & t>0, x \in \mathbb{R}^{n} \\ { }^{C} D_{0 \mid t}^{\gamma_{2}} v-\Delta v=g(u(t, \cdot)), & t>0, x \in \mathbb{R}^{n}\end{cases}
$$

subject to the initial conditions

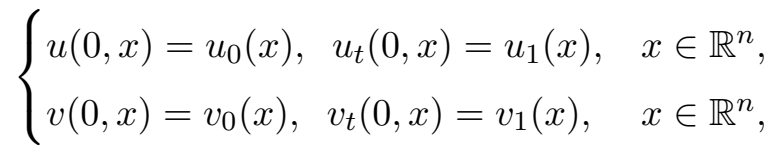

where $1<\gamma_{1}, \gamma_{2}<2,{ }^{C} D_{0 \mid t}^{\alpha} u$ denotes the Caputo derivative, defined for a function $u$ of class $C^{2}$, as (see, e.g., 31)

$$
\left({ }^{C} D_{0 \mid t}^{\alpha} u\right)(t):=\frac{1}{\Gamma(2-\alpha)} \int_{0}^{t} \frac{u_{t t}(s, \cdot)}{(t-s)^{\alpha-1}} d s, \quad 1<\alpha<2,
$$

$\Delta$ is the Laplacian, $f(v)= \pm|v|^{p-1} v$ or $\pm|v|^{p}, g(u)= \pm|u|^{q-1} u$ or $\pm|u|^{q}, p, q \geq 1$, and $u_{0}$, $v_{0}, u_{1}, v_{1}$ are given initial data.

Observe that system (1.1) interpolates reaction-diffusion system $\left(\gamma_{1}=\gamma_{2}=1\right)$ and hyperbolic system $\left(\gamma_{1}=\gamma_{2}=2\right)$.

Received May 25, 2020; Accepted August 30, 2021.

Communicated by Cheng-Hsiung Hsu.

2020 Mathematics Subject Classification. 35J05, 26A33, 35L15.

Key words and phrases. coupled fractional-wave equations, polynomial nonlinearities, global solution, blow-up.

*Corresponding author. 
Before we present our results and comment on them, let us dwell on some related existing results.

Escobedo and Herrero [13] studied the global existence and blowing-up solutions of the system

$$
\begin{cases}u_{t}-\Delta u=v^{p}, & t>0, x \in \mathbb{R}^{N}, \\ v_{t}-\Delta v=u^{q}, & t>0, x \in \mathbb{R}^{N} .\end{cases}
$$

In particular, for

$$
p q>1, \quad \frac{N}{2} \leq \frac{\max \{p, q\}+1}{p q-1},
$$

they have shown that every nontrivial solution of $(1.3)$ blows-up in a finite time $T^{*}=$ $T^{*}(u, v)$, and

$$
\limsup _{t \rightarrow T^{*}}\|u(t)\|_{\infty}=\limsup _{t \rightarrow T^{*}}\|v(t)\|_{\infty}=+\infty .
$$

Some related results concerning global existence or blowing-up solutions can be found in [14, 27-30], etc. In particular, see the review papers [4,11] and the authoritative paper [26].

Blowing-up solutions and global solutions for time-fractional differential systems have been studied, for example, in $1,3,12,16,18,20,22,34$.

Concerning the system of wave equations

$$
\begin{cases}u_{t t}-\Delta u=|v|^{p}, & 0<t<T, x \in \mathbb{R}^{N}, \\ v_{t t}-\Delta v=|u|^{q}, & 0<t<T, x \in \mathbb{R}^{N},\end{cases}
$$

subject to initial data

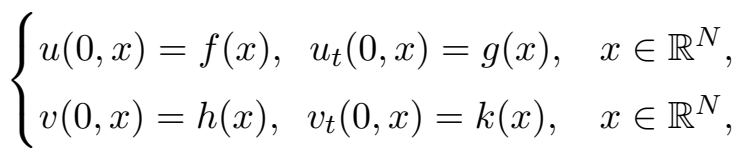

where $f, g, h, k \in C_{0}^{\infty}\left(R^{N}\right)$, we may mention the works [8 10]. For $N=3$ in [8], the following optimal results were obtained:

$\triangleright$ If $p, q>1$ and

$$
\max \left\{\frac{p+2+q^{-1}}{p q-1}, \frac{q+2+p^{-1}}{p q-1}\right\}>1,
$$

then the classical solution to (1.4) -1.5 blows-up in a finite time.

$\triangleright$ If $p, q>1$ and

$$
\max \left\{\frac{p+2+q^{-1}}{p q-1}, \frac{q+2+p^{-1}}{p q-1}\right\}<1,
$$

then there exists a global classical solution to (1.4)- 1.5 for sufficiently "small" initial data. 
Our interest in (1.1) stems from the fact that it interpolates different situations; for example, reaction-diffusion systems with fractional derivatives can model chemical reactions taking place in porous media. In this case, fractional (nonlocal) terms with order in $(0,1)$ account for the anomalous diffusion [23,25]. Experimental results show that several complex systems have a non-local dynamics.

On the other hand, equations/systems of fractional differential equations with order in $(1,2)$ have been studied in 7, 24, 33, etc. Examples include mechanical, acoustical, biological phenomena, marine sediments, etc. [19, 32].

In the present paper, we consider the problem (1.1) 1.2 and present conditions, relating the space dimension $N$ with the parameters $\gamma_{1}, \gamma_{2}, p$, and $q$, for which the solution of (1.1)-1.2 exists globally in time and satisfies $L^{\infty}$-decay estimates. We also investigate blowing-up in finite time solutions with initial data having positive average. Our study of global existence employs the mild formulation of the solution via Mittag-Leffler's function, while we use the test function approach due to Mitidieri and Pohozaev [26] for the case of blowing-up solutions. The test function approach has been used by several authors, (for instance, see $[5,6,15,22,27,34)$. To the best of our knowledge, there do not exist global existence and large time behavior results for the time-fractional diffusion system with two different fractional powers. Thus our results are new and contribute significantly to the existing literature on the topic.

The rest of this paper is organized as follows. In next section, we present some preliminary lemmas, basic facts and useful tools such as time fractional derivative, $L^{p}-L^{q}$ estimates of the fundamental solution of the linear time fractional wave equation. Section 3 contains the main results of the paper. Finally, Section 4 is devoted to the proof of small data global existence and blow-up in finite time of the solution of problem (1.1) - 1.2).

In the sequel, $C$ will be a positive constant which may have different values from line to line. The space $L^{p}\left(\mathbb{R}^{N}\right)(1 \leq p<\infty)$ will be equipped with the norm:

$$
\|u\|_{L^{p}\left(\mathbb{R}^{N}\right)}^{p}=\int_{\mathbb{R}^{N}}|u(t, x)|^{p} d x
$$

\section{Preliminaries}

The Riemann-Liouville fractional integral of order $0<\alpha<1$ of $f(t) \in L^{1}(0, T)$ is defined as

$$
\left(J_{0 \mid t}^{\alpha} f\right)(t)=\frac{1}{\Gamma(\alpha)} \int_{0}^{t}(t-\tau)^{\alpha-1} f(\tau) d \tau,
$$

where $\Gamma$ stands for the usual Euler gamma function.

The left-sided Riemann-Liouville derivative $D_{0 \mid t}^{\alpha} f$ (see $[31]$ ), for $f \in C^{m-1}(0, T)$, of 
order $\alpha$ is defined as follows:

$$
\left(D_{0 \mid t}^{\alpha} f\right)(t)=\frac{d^{m}}{d t^{m}}\left(J_{0 \mid t}^{m-\alpha} f\right)(t), \quad t>0, m-1<\alpha<m, m \in \mathbb{N} .
$$

The Caputo fractional derivative of a function $f \in C^{m}(0, T)$ is defined as

$$
\left({ }^{C} D_{0 \mid t}^{\alpha} f\right)(t)=J_{0 \mid t}^{m-\alpha} f^{(m)}(t), \quad t>0, m-1<\alpha<m, m \in \mathbb{N} .
$$

For $0<\alpha<1$ and $f$ of class $C^{1}$, we have

$$
\left(D_{0 \mid t}^{\alpha} f\right)(t)=\frac{1}{\Gamma(1-\alpha)}\left[\frac{f(0)}{t^{\alpha}}+\int_{0}^{t} \frac{f^{\prime}(\sigma)}{(t-\sigma)^{\alpha}} d \sigma\right],
$$

and

$$
\left(D_{t \mid T}^{\alpha} f\right)(t)=\frac{1}{\Gamma(1-\alpha)}\left[\frac{f(T)}{(T-t)^{\alpha}}-\int_{t}^{T} \frac{f^{\prime}(\sigma)}{(\sigma-t)^{\alpha}} d \sigma\right] .
$$

The Caputo derivative is related to the Riemann-Liouville derivative for $f \in A C[0, T]$ (the space of absolutely continuous functions defined on $[0, T]$ ) by

$$
\left({ }^{C} D_{0 \mid t}^{\alpha} f\right)(t)=D_{0 \mid t}^{\alpha}(f(t)-f(0))
$$

Assume that $0<\alpha<1, f \in C^{1}([a, b])$ and $g \in C(a, b)$. Then the formula of integration by parts is

$$
\int_{a}^{b} f(t)\left(D_{0 \mid t}^{\alpha} g\right)(t) d t=\int_{a}^{b} g(t)\left({ }^{C} D_{t \mid T}^{\alpha} f\right)(t) d t+\left.f(a)\left(I_{a \mid t}^{1-\alpha} g\right)(t)\right|_{t=a} ^{t=b}
$$

The Mittag-Leffler function is defined (see [31]) by

$$
E_{\alpha, \beta}(z)=\sum_{k=0}^{\infty} \frac{z^{k}}{\Gamma(\alpha k+\beta)}, \quad \alpha, \beta \in \mathbb{C}, \Re(\alpha)>0, z \in \mathbb{C}
$$

its Riemann-Liouville fractional integral satisfies

$$
J_{0 \mid t}^{1-\alpha}\left(t^{\alpha-1} E_{\alpha, \alpha}\left(\lambda t^{\alpha}\right)\right)=E_{\alpha, 1}\left(\lambda t^{\alpha}\right) \quad \text { for } \lambda \in \mathbb{C}, 0<\alpha<1
$$

For later use, let

$$
\varphi(t)=\left(1-\frac{t}{T}\right)_{+}^{l}, \quad l \geq 2
$$

then

$$
{ }^{C} D_{t \mid T}^{\alpha} \varphi(t)=\frac{\Gamma(l+1)}{\Gamma(l+1-\alpha)} T^{-\alpha}\left(1-\frac{t}{T}\right)_{+}^{l-\alpha}, \quad t \leq T,
$$

(see, for example, 22]). 


\subsection{Linear estimates}

In this section, we present fundamental estimates which will be used to prove Theorem 3.3 . For $1<\alpha<2$, we define the operators $\widetilde{E}_{\alpha, 1}(t, x)$ and $\widetilde{E}_{\alpha, \alpha}(t, x)$ as follows:

$$
\begin{array}{lll}
\widetilde{E}_{\alpha, 1}(t, x)=(2 \pi)^{-N / 2} \mathcal{F}^{-1}\left(E_{\alpha, 1}\left(-4 \pi^{2} t^{\alpha}|\xi|^{2}\right)\right)(x), & & x \in \mathbb{R}^{N}, t>0, \\
\widetilde{E}_{\alpha, 2}(t, x)=(2 \pi)^{-N / 2} \mathcal{F}^{-1}\left(E_{\alpha, 2}\left(-4 \pi^{2} t^{\alpha}|\xi|^{2}\right)\right)(x), & & x \in \mathbb{R}^{N}, t>0, \\
\widetilde{E}_{\alpha, \alpha}(t, x)=(2 \pi)^{-N / 2} \mathcal{F}^{-1}\left(E_{\alpha, \alpha}\left(-|\xi|^{2} t^{\alpha}\right)\right)(x), & x \in \mathbb{R}^{N}, t>0 .
\end{array}
$$

Consider the following linear inhomogeneous time fractional equation with initial data:

$$
\begin{cases}{ }^{C} D_{0 \mid t}^{\alpha} u-\Delta u=f(t, x), & t>0, x \in \mathbb{R}^{N}, 1<\alpha<2, \\ u(0, x)=u_{0}(x), \quad u_{t}(0, x)=u_{1}(x), & x \in \mathbb{R}^{N} .\end{cases}
$$

If $u_{0} \in S\left(R^{N}\right)$ (the Schwartz space), $u_{1} \in S\left(R^{N}\right)$ and $f \in L^{1}\left((0,+\infty), \mathcal{S}\left(\mathbb{R}^{N}\right)\right)$, then by [18] (see also [1]) problem (2.1) admits a solution $u \in C^{\alpha}\left([0,+\infty) ; \mathcal{S}\left(\mathbb{R}^{N}\right)\right.$ ), which satisfies

$$
u(t, x)=\widetilde{E}_{\alpha, 1}(t, x) u_{0}(x)+t \widetilde{E}_{\alpha, 2}(t, x) u_{1}(x)+\int_{0}^{t}(t-s)^{\alpha-1} \widetilde{E}_{\alpha, \alpha}(t-s) f(s, x) d s,
$$

where $\widetilde{E}_{\alpha, \beta}(t, \cdot) h,(\beta=1,2, \alpha)$ is defined for $h \in S^{\prime}\left(R^{N}\right)$ by

$$
\widetilde{E}_{\alpha, \beta}(t, \cdot) h:=\widetilde{E}_{\alpha, \beta}(t, \cdot) * h(x)=\int_{\mathbb{R}^{N}} \widetilde{E}_{\alpha, \beta}(t, x-y) h(y) d y .
$$

The following lemmas contain the so called smoothing effect of the Mittag-Leffler operators family $\left\{\widetilde{E}_{\alpha, 1}(t)\right\}_{t \geq 0}$ and $\left\{\widetilde{E}_{\alpha, \alpha}(t)\right\}_{t \geq 0}$ in Lebesgue spaces and play an important role in obtaining the first result of this paper; they appear in [18, Lemma 5.1] and 3, Lemma 5.1]. Their proofs are based on the Fourier multiplier theorem combined with a scaling argument (see [2, Lemma 3.1-(i)] or [3, Propositions 4.2 and 4.3]).

Lemma 2.1. 3, Lemma 5.1] Let $1<p_{1} \leq p_{2}<\infty, 1<\alpha<2$ and $\lambda=\frac{N}{p_{1}}-\frac{N}{p_{2}}$. Then there is a constant $C>0$ such that

$$
\begin{array}{ll}
\left\|\widetilde{E}_{\alpha, 1}(t) f\right\|_{L^{p_{2}}} \leq C t^{-\frac{\alpha}{2} \lambda}\|f\|_{L^{p_{1}}} & \text { if } \lambda<2, \\
\left\|t \widetilde{E}_{\alpha, 2}(t) f\right\|_{L^{p_{2}}} \leq C t^{1-\frac{\alpha}{2} \lambda}\|f\|_{L^{p_{1}}} & \text { if } \frac{2}{\alpha}<\lambda<2, \\
\left\|t \widetilde{E}_{\alpha, 2}(t) f\right\|_{L^{p_{2}}} \leq C t^{-\frac{\alpha}{2} \lambda}\|f\|_{\dot{\mathcal{H}}_{p_{1}}^{-\frac{2}{\alpha}}} & \text { if } \frac{2}{\alpha}<\lambda<2, \\
\left\|\widetilde{E}_{\alpha, \alpha}(t) f\right\|_{L^{p_{2}}} \leq C t^{-\frac{\alpha}{2} \lambda}\|f\|_{L^{p_{1}}} & \text { if }\left(2-\frac{2}{\alpha}\right)<\lambda<2
\end{array}
$$

for all $f \in S^{\prime}\left(R^{N}\right)$, where $\dot{H}_{p_{1}}^{-\frac{2}{\alpha}}$ is the homogeneous Sobolev spaces of negative order $-\frac{2}{\alpha}$. 
Lemma 2.2. The family of operators $\left\{\widetilde{E}_{\alpha, 1}(t)\right\}_{t>0},\left\{\widetilde{E}_{\alpha, 1}(t)\right\}_{t>0}$ and $\left\{\widetilde{E}_{\alpha, \alpha}(t)\right\}_{t>0}$ enjoy the following $L^{p_{1}}-L^{p_{1}}$ estimates property:

(i) If $h \in L^{p_{1}}\left(\mathbb{R}^{N}\right)\left(1 \leq p_{1} \leq+\infty\right)$, then $\widetilde{E}_{\alpha, \beta}(t) h \in L^{p_{1}}\left(\mathbb{R}^{N}\right)$ and

$$
\left\|\widetilde{E}_{\alpha, \beta}(t) h\right\|_{L^{p_{1}\left(\mathbb{R}^{N}\right)}} \leq C\|h\|_{L^{p_{1}\left(\mathbb{R}^{N}\right)}}, \quad t>0 \quad \text { for } \beta=1,2, \alpha
$$

for some positive constant $C>0$.

(ii) Let $p_{1}>N / 2$. If $h \in L^{p_{1}}\left(\mathbb{R}^{N}\right)$, then $\widetilde{E}_{\alpha, \beta}(t) h \in L^{\infty}\left(\mathbb{R}^{N}\right)$ and we have

$$
\left\|\widetilde{E}_{\alpha, \beta}(t) h\right\|_{L^{\infty}\left(\mathbb{R}^{N}\right)} \leq C t^{-\frac{\alpha}{2} \frac{N}{p_{1}}}\|h\|_{L^{p_{1}\left(\mathbb{R}^{N}\right)}}, \quad t>0 \quad \text { for } \beta=1,2, \alpha .
$$

Proof. We use the following pointwise estimates that are shown in 21, Theorem 5.1]:

$$
\left|\widetilde{E}_{\alpha, \alpha}(t, x)\right| \leq|x|^{-N} \exp \left\{-c\left(t^{-\alpha}|x|^{2}\right)^{\frac{1}{2-\alpha}}\right\} \quad \text { if } R:=|x|^{2} t^{-\alpha} \geq 1
$$

and if $R:=|x|^{2} t^{-\alpha}<1$, then we have

$$
\left|\widetilde{E}_{\alpha, \alpha}(t, x)\right| \leq \begin{cases}t^{-\frac{\alpha N}{2}}, & N<2 \\ t^{-\alpha}|x|^{-N+2}\left(1+\left|\ln \left(|x|^{2} t^{-\alpha}\right)\right|\right), & N=2 \\ |x|^{-N+2} t^{-\alpha}, & N>2\end{cases}
$$

Concerning the operator $t \widetilde{E}_{\alpha, 2}(t)$, we have the pointwise estimates

$$
\left|t \widetilde{E}_{\alpha, 2}(t)\right| \leq C|x|^{-N} t \exp \left\{-c\left(t^{-\alpha}|x|^{2}\right)^{\frac{1}{2-\alpha}}\right\} \quad \text { if } R:=|x|^{2} t^{-\alpha} \geq 1,
$$

and if $R:=|x|^{2} t^{-\alpha}<1$, then

$$
\left|t \widetilde{E}_{\alpha, 2}(t, x)\right| \leq \begin{cases}t^{1-\frac{\alpha N}{2}}, & N<2, \\ |x|^{-N+2} t^{1-\alpha}\left(1+\left|\ln \left(|x|^{2} t^{-\alpha}\right)\right|\right), & N=2, \\ |x|^{-N+2} t^{1-\alpha}, & N>2 .\end{cases}
$$

Arguing as in Zacher et al. 20, $\widetilde{E}_{\alpha, 1}(t, \cdot), \widetilde{E}_{\alpha, 2}(t, \cdot)$ and $\widetilde{E}_{\alpha, \alpha}(t, \cdot)$ are Lebesgue integrable.

In fact, we have

$$
\int_{\mathbb{R}^{N}}\left|\widetilde{E}_{\alpha, \alpha}(t, x)\right| d x=\int_{\{R \geq 1\}}\left|\widetilde{E}_{\alpha, \alpha}(t, x)\right| d x+\int_{\{R<1\}}\left|\widetilde{E}_{\alpha, \alpha}(t, x)\right| d x .
$$

Using the first pointwise estimate, we get

$$
\begin{aligned}
\int_{\{R \geq 1\}}\left|\widetilde{E}_{\alpha, \alpha}(t, x)\right| d x & \leq \int_{\{R \geq 1\}}|x|^{-N} \exp \left\{-c\left(t^{-\alpha}|x|^{2}\right)^{\frac{1}{2-\alpha}}\right\} d x \\
& =\int_{t^{\frac{\alpha}{2}}}^{+\infty} r^{-N} \exp \left\{-c\left(t^{-\alpha} r^{2}\right)^{\frac{1}{2-\alpha}}\right\} r^{N-1} d r \\
& =\int_{t^{\frac{\alpha}{2}}}^{+\infty} r^{-1} \exp \left\{-c\left(t^{-\alpha} r^{2}\right)^{\frac{1}{2-\alpha}}\right\} d r, \quad \text { set } z=t^{-\frac{\alpha}{2}} r \\
& =\int_{1}^{+\infty} z^{-1} \exp \left\{-c\left(z^{2}\right)^{\frac{1}{2-\alpha}}\right\} d z \leq C
\end{aligned}
$$


On the other hand, if $N<2$, we have

$$
\int_{\{R \leq 1\}}\left|\widetilde{E}_{\alpha, \alpha}(t, x)\right| d x \leq \int_{\{R \leq 1\}} t^{-\frac{\alpha N}{2}} d x=t^{-\frac{\alpha N}{2}} \int_{0}^{t^{\frac{\alpha}{2}}} r^{N-1} d r=t^{-\frac{\alpha N}{2}} \frac{t^{\frac{\alpha N}{2}}}{N}=C .
$$

For $N=2$, we have

$$
\begin{aligned}
\int_{\{R \leq 1\}}\left|\widetilde{E}_{\alpha, \alpha}(t, x)\right| d x & \leq \int_{\{R \leq 1\}}|x|^{-N+2} t^{-\alpha}\left(1-\ln \left(|x|^{2} t^{-\alpha}\right)\right) d x \\
& =t^{-\alpha} \int_{0}^{t^{\frac{\alpha}{2}}}\left(1+\left|\ln \left(r^{2} t^{-\alpha}\right)\right|\right) r d r=t^{-\alpha} t^{\frac{\alpha}{2}} \int_{0}^{1}\left(1-\ln \left(z^{2}\right)\right) z d z=C .
\end{aligned}
$$

When $N>2$, we have

$$
\begin{aligned}
\int_{\{R \leq 1\}}\left|\widetilde{E}_{\alpha, \alpha}(t, x)\right| d x & \leq \int_{\{R \leq 1\}}|x|^{-N+2} t^{-\alpha} d x \\
& =t^{-\alpha} \int_{0}^{t^{\frac{\alpha}{2}}} r^{-N+2} r^{N-1} d r=t^{-\alpha} \int_{0}^{t^{\frac{\alpha}{2}}} r d r,
\end{aligned}
$$

SO

$$
\int_{\{R \leq 1\}}\left|\widetilde{E}_{\alpha, \alpha}(t, x)\right| d x \leq \frac{1}{2} .
$$

The first result (i) follows from Young's convolution inequality, that is,

$$
\begin{aligned}
\left\|\widetilde{E}_{\alpha, \alpha}(t, \cdot) h\right\|_{L^{p_{1}\left(\mathbb{R}^{N}\right)}} & =\left\|\widetilde{E}_{\alpha, \alpha}(t, \cdot) * h(x)\right\|_{L^{p_{1}\left(\mathbb{R}^{N}\right)}} \\
& \leq\left\|\widetilde{E}_{\alpha, \alpha}(t, \cdot)\right\|_{L^{1}\left(\mathbb{R}^{N}\right)}\|h\|_{L^{p_{1}\left(\mathbb{R}^{N}\right)}} \leq C\|h\|_{L^{p_{1}\left(\mathbb{R}^{N}\right)}} .
\end{aligned}
$$

In a s similar manner, it can be shown that the operators $\widetilde{E}_{\alpha, 1}(t, \cdot)$ and $\widetilde{E}_{\alpha, 2}(t, \cdot)$ are bounded.

In order to show statement (ii), we need to prove that $\widetilde{E}_{\alpha, \alpha}(t, \cdot)$, belongs to $L^{p_{2}}\left(\mathbb{R}^{N}\right)$

$$
\begin{aligned}
\int_{\{R \geq 1\}}\left|\widetilde{E}_{\alpha, \alpha}(t, x)\right|^{p_{2}} d x & \leq \int_{\{R \geq 1\}}|x|^{-N p_{2}} \exp \left\{-c\left(t^{-\alpha}|x|^{2}\right)^{\frac{1}{2-\alpha}}\right\} d x \\
& =\int_{t^{\frac{\alpha}{2}}}^{+\infty} r^{-N p_{2}} \exp \left\{-c\left(t^{-\alpha} r^{2}\right)^{\frac{1}{2-\alpha}}\right\} r^{N-1} d r \\
& =\int_{t^{\frac{\alpha}{2}}}^{+\infty} r^{-N r+N-1} \exp \left\{-c\left(t^{-\alpha} r^{2}\right)^{\frac{1}{2-\alpha}}\right\} d r, \quad \text { set } z=t^{-\frac{\alpha}{2}} r \\
& =t^{-\frac{\alpha}{2} N\left(p_{2}-1\right)} \int_{1}^{+\infty} z^{-N p_{2}+N-1} \exp \left\{-c\left(z^{2}\right)^{\frac{1}{2-\alpha}}\right\} d z \\
& \leq C t^{-\frac{\alpha}{2} N\left(p_{2}-1\right)} .
\end{aligned}
$$

On the other hand, if $N=1$, we have

$$
\begin{aligned}
\int_{\{R \leq 1\}}\left|\widetilde{E}_{\alpha, \alpha}(t, x)\right|^{p_{2}} d x & \leq \int_{\{R \leq 1\}} t^{-\frac{\alpha N}{2} p_{2}} d x=t^{-\frac{\alpha N}{2} p_{2}} \int_{0}^{t^{\frac{\alpha}{2}}} r^{N-1} d r \\
& =\frac{1}{N} t^{-\frac{\alpha N}{2} p_{2}+\frac{\alpha}{2}}=C t^{-\frac{\alpha}{2} N\left(p_{2}-1\right)}
\end{aligned}
$$


For $N=2$, we have

$$
\begin{aligned}
\int_{\{R \leq 1\}}\left|\widetilde{E}_{\alpha, \alpha}(t, x)\right|^{p_{2}} d x & \leq \int_{\{R \leq 1\}} t^{-\alpha p_{2}}\left(1-\ln \left(|x|^{2} t^{-\alpha}\right)\right)^{p_{2}} d x \\
& =t^{-\alpha p_{2}} \int_{0}^{t^{\frac{\alpha}{2}}}\left(1+\left|\ln \left(r^{2} t^{-\alpha}\right)\right|\right)^{p_{2}} r^{N-1} d r \\
& =t^{-\alpha\left(p_{2}-1\right)} \int_{0}^{1}\left(1-\ln \left(z^{2}\right)\right)^{p_{2}} z d z=C t^{-\alpha\left(p_{2}-1\right)} .
\end{aligned}
$$

When $N>2$, we have

$$
\begin{aligned}
\int_{\{R \leq 1\}}\left|\widetilde{E}_{\alpha, \alpha}(t, x)\right|^{p_{2}} d x & \leq \int_{\{R \leq 1\}}|x|^{-(N-2) p_{2}} t^{-\alpha p_{2}} d x \\
& =t^{-\alpha p_{2}} \int_{0}^{t^{\frac{\alpha}{2}}} r^{-(N-2) p_{2}} r^{N-1} d r=t^{-\alpha p_{2}} \int_{0}^{t^{\frac{\alpha}{2}}} r^{-(N-2) p_{2}+N-1} d r
\end{aligned}
$$

provided $N>(N-2) p_{2}$. So

$$
\int_{\{R \leq 1\}}\left|\widetilde{E}_{\alpha, \alpha}(t, x)\right|^{p_{2}} d x \leq C t^{-\alpha p_{2}-\frac{\alpha}{2}(N-2) p_{2}+\frac{\alpha}{2} N}=C t^{-\frac{\alpha}{2} N\left(p_{2}-1\right)} .
$$

Hence $\left\|\widetilde{E}_{\alpha, \alpha}(t, \cdot)\right\|_{p_{2}} \leq C t^{-\frac{\alpha}{2} N\left(1-\frac{1}{p_{2}}\right)}$, for $p_{2}<N /(N-2)$.

Now (ii) follows by Young's convolution inequality and the last estimate

$$
\left\|\widetilde{E}_{\alpha, \alpha}(t, \cdot) * f\right\|_{L^{\infty}} \leq\left\|\widetilde{E}_{\alpha, \alpha}(t, \cdot)\right\|_{L^{p_{1}^{\prime}}}\|f\|_{L^{p_{1}}} \leq C t^{-\frac{\alpha}{2} \frac{N}{p_{1}}}\|f\|_{L^{p_{1}}} \quad \text { for } p_{1}>\frac{N}{2}
$$

where $p_{1}^{\prime}$ is the conjugate of $p_{1}\left(1 / p_{1}+1 / p_{1}^{\prime}=1\right)$. Arguing in a similar way, we obtain $L^{p_{1}}-L^{\infty}$ estimates to the operators $\widetilde{E}_{\alpha, \beta}(t)$ for $\beta=1,2$.

Lemma 2.3. Let $l \geq 1$, and let the function $f(t, x)$ satisfy

$$
\|f(t, \cdot)\|_{l} \leq C_{1}, \quad 0 \leq t \leq 1, \quad\|f(t, \cdot)\|_{l} \leq C_{2} t^{-\alpha}, \quad t>0
$$

for some positive constants $C_{1}, C_{2}$ and $\alpha$. Then

$$
\|f(t, \cdot)\|_{l} \leq \max \left\{C_{1}, C_{2}\right\}(1+t)^{-\beta} \quad \text { for all } 0<\beta \leq \alpha \text { and } t \geq 0
$$

\section{Main results}

In this section, we state our main results. Let us begin with the definition of a mild solution of problem $1.1-1.2$. 
Definition 3.1. Let $u_{0}, v_{0}, u_{1}, v_{1} \in X,\left(X:=L^{1}\left(\mathbb{R}^{N}\right) \cap L^{\infty}\left(\mathbb{R}^{N}\right)\right), 1<\gamma_{1}, \gamma_{2}<2$, $f, g \in L^{1}\left((0, T), \mathcal{S}\left(\mathbb{R}^{N}\right)\right)$ and $T>0$. We call $(u, v) \in C([0, T] ; \mathbb{X}) \times C([0, T] ; \mathbb{X})$ a mild solution of system (1.1)-(1.2) if $(u, v)$ satisfies the following integrals

$$
\begin{aligned}
u(t, x)= & \widetilde{E}_{\gamma_{1}, 1}(t, x) u_{0}(x)+t \widetilde{E}_{\gamma_{1}, 2}(t, x) u_{1}(x) \\
& +\int_{0}^{t}(t-\tau)^{\gamma_{1}-1} \widetilde{E}_{\gamma_{1}, \gamma_{1}}(t-\tau, x) f(v(\tau, x)) d \tau, \\
v(t, x)= & \widetilde{E}_{\gamma_{2}, 1}(t, x) v_{0}(x)+t \widetilde{E}_{\gamma_{2}, 2}(t, x) v_{1}(x) \\
& +\int_{0}^{t}(t-\tau)^{\gamma_{1}-1} \widetilde{E}_{\gamma_{2}, \gamma_{2}}(t-\tau, x) g(u(\tau, x)) d \tau .
\end{aligned}
$$

The existence and uniqueness of a local solution of 1.1 can be established by using the Banach fixed point theorem and Gronwall's inequality.

Proposition 3.2 (Local existence of a mild solution). Let $u_{0}, v_{0}, u_{1}, v_{1} \in X, 1<\gamma_{1}, \gamma_{2}<$ $2, p, q \geq 1$ such that $p q>1$. Then there exist a maximal time $T_{\max }>0$ and a unique mild solution to problem $1.1-(1.2)$, such that either

(i) $T_{\max }=\infty$ (the solution is global), or

(ii) $T_{\max }<\infty$ and $\lim _{t \rightarrow T_{\max }}\left(\|u(t)\|_{\infty}+\|v(t)\|_{\infty}\right)=\infty$ (the solution blows up in a finite time).

Moreover, for any $s_{1}, s_{2} \in(1,+\infty),(u, v) \in C\left([0, T] ; L^{s_{1}}\left(\mathbb{R}^{N}\right) \times L^{s_{2}}\left(\mathbb{R}^{N}\right)\right)$.

Now, we are in a position to state the first main result of this section concerning global existence and large time behavior of solutions of $(1.1)-(1.2)$.

Theorem 3.3 (Global existence of a mild solution). Let $N \geq 2, q \geq p \geq 1, p q>1$, $1<\gamma_{1} \leq \gamma_{2}<2$. If

$$
\frac{N}{2} \geq \max \left\{\frac{1}{\gamma_{1}}+\frac{q+1}{p q-1}, \frac{1}{\gamma_{1}}+\frac{p \gamma_{2}+\gamma_{1}}{\gamma_{1}(p q-1)}\right\}
$$

the initial data satisfy

$$
\left\|u_{0}\right\|_{\mathbb{X}}+\left\|u_{1}\right\|_{\mathbb{X}}+\left\|v_{0}\right\|_{\mathbb{X}}+\left\|v_{1}\right\|_{\mathbb{X}} \leq \varepsilon_{0}
$$

for some $\varepsilon_{0}>0$, then problem $(1.1)-(1.2)$ admits a global mild solution and that

$$
\begin{aligned}
& u \in L^{\infty}\left([0, \infty), L^{\infty}\left(\mathbb{R}^{N}\right)\right) \cap L^{\infty}\left([0, \infty), L^{s_{1}}\left(\mathbb{R}^{N}\right)\right), \\
& v \in L^{\infty}\left([0, \infty), L^{\infty}\left(\mathbb{R}^{N}\right)\right) \cap L^{\infty}\left([0, \infty), L^{s_{2}}\left(\mathbb{R}^{N}\right)\right),
\end{aligned}
$$

where $s_{1}>q$ and $s_{2}>p$. 
Furthermore, for any $\delta$ satisfying $1-\frac{1+q}{(p+1) q \gamma_{2}}<\delta<\min \left\{1, \frac{N(p q-1)}{2 q(p+1)}\right\}$,

$$
\begin{array}{cl}
\|u(t)\|_{s_{1}} \leq C(t+1)^{-\frac{(1-\delta)\left(\gamma_{1}+p \gamma_{2}\right)}{p q-1}}, & t \geq 0, \\
\|v(t)\|_{s_{2}} \leq C(t+1)^{-\frac{(1-\delta)\left(\gamma_{2}+q \gamma_{1}\right)}{p q-1}}, & t \geq 0 .
\end{array}
$$

If, in addition,

$$
\frac{p N}{2 s_{2}}<1 \quad \text { and } \quad \frac{q N}{2 s_{1}}<1
$$

or

$$
N>2, \quad \frac{p N}{2 s_{2}}<1 \quad \text { and } \quad \frac{q N}{2 s_{1}} \geq 1
$$

or

$$
N>2, \quad \frac{q N}{2 s_{1}} \geq 1, \quad \frac{p N}{2 s_{2}} \geq 1 \quad \text { and } \quad q \geq p>1 \quad \text { with } \sqrt{\frac{(p+1) q \gamma_{1}}{(q+1) p}}<\gamma_{1} \leq \gamma_{2}<2,
$$

then

$$
\begin{gathered}
u, v \in L^{\infty}\left([0, \infty), L^{\infty}\left(\mathbb{R}^{N}\right)\right), \\
\|u(t)\|_{\infty} \leq C(t+1)^{-\widetilde{\sigma}}, \quad\|v(t)\|_{\infty} \leq C(t+1)^{-\widehat{\sigma}} \quad \text { for all } t \geq 0
\end{gathered}
$$

for some positive constants $\widetilde{\sigma}$ and $\widehat{\sigma}$.

Definition 3.4 (Weak solution). Let $u_{0}, v_{0} \in L_{\mathrm{loc}}^{\infty}\left(\mathbb{R}^{N}\right), u_{1}, v_{1} \in L_{\mathrm{loc}}^{\infty}\left(\mathbb{R}^{N}\right), T>0$. We say that $(u, v) \in L^{q}\left((0, T), L_{\text {loc }}^{\infty}\left(\mathbb{R}^{N}\right)\right) \times L^{p}\left((0, T), L_{\text {loc }}^{\infty}\left(\mathbb{R}^{N}\right)\right)$ is a weak solution of 1.1 - 1.2 if for all nonnegative test functions $\varphi \in C_{t, x}^{1,2}\left([0, T] \times \mathbb{R}^{N}\right)$ with compact support, such that $\varphi(T, \cdot)=0$, the system of integral equalities

$$
\begin{aligned}
& \int_{0}^{T} \int_{\mathbb{R}^{N}} u D_{t \mid T}^{\gamma_{1}} \varphi(t, x) d x d t-\int_{0}^{T} \int_{\mathbb{R}^{N}} u \Delta \varphi(t, x) d x d t \\
= & \int_{\mathbb{R}^{N}} u_{0}(x)\left(D_{t \mid T}^{\gamma_{1}-1} \varphi\right)(0, \cdot) d x+\int_{0}^{T} \int_{\mathbb{R}^{N}} u_{1} D_{t \mid T}^{\gamma_{1}-1} \varphi(t, x) d x d t \\
& +\int_{0}^{T} \int_{\mathbb{R}^{N}} f(v(\tau, x)) \varphi(t, x) d x d t \\
& \int_{0}^{T} \int_{\mathbb{R}^{N}} v D_{t \mid T}^{\gamma_{2}} \varphi(t, x) d x d t-\int_{0}^{T} \int_{\mathbb{R}^{N}} v \Delta \varphi(t, x) d x d t \\
= & \int_{\mathbb{R}^{N}} v_{0}(x)\left(D_{t \mid T}^{\gamma_{2}-1} \varphi\right)(0, \cdot) d x+\int_{0}^{T} \int_{\mathbb{R}^{N}} v_{1} D_{t \mid T}^{\gamma_{2}-1} \varphi(t, x) d x d t \\
& +\int_{0}^{T} \int_{\mathbb{R}^{N}} g(u(\tau, x)) \varphi(t, x) d x d t
\end{aligned}
$$

holds. 
Similar to the proof in [15], we can obtain the following lemma asserting that the mild solution is the weak solution.

Lemma 3.5. Assume that $\left(u_{0}, v_{0}\right),\left(u_{1}, v_{1}\right) \in S\left(\mathbb{R}^{N}\right) \times S\left(\mathbb{R}^{N}\right)$ and let $(u, v) \in C^{\gamma_{1}}([0, T]$, $\left.\mathcal{S}\left(\mathbb{R}^{N}\right)\right) \times C^{\gamma_{2}}\left([0, T], \mathcal{S}\left(\mathbb{R}^{N}\right)\right)$ be a mild solution of $\left.11.1-1.2\right)$. Then $(u, v)$ is also a weak solution of 1.1$)-1.2$.

Proof. As $(u, v)$ is a mild solution, we have

$$
u(t, x)=\widetilde{E}_{\gamma_{1}, 1}(t, x) u_{0}(x)+t \widetilde{E}_{\gamma_{1}, 2}(t, x) u_{1}(x)+\int_{0}^{t}(t-s)^{\gamma_{1}-1} \widetilde{E}_{\gamma_{1}, \gamma_{1}}(t-s) f(v(s, x)) d s .
$$

Differentiating with respect to $t$ and noting that $1<\gamma_{1}<2$, we get

$$
\begin{aligned}
u_{t}(t, x)-u_{1}(x)= & \partial_{t} \widetilde{E}_{\gamma_{1}, 1}(t, x) u_{0}(x)+\partial_{t}\left(t \widetilde{E}_{\gamma_{1}, 2}(t, x)\right) u_{1}(x)-u_{1}(x) \\
& +\int_{0}^{t}(t-s)^{\gamma_{1}-2} \widetilde{E}_{\gamma_{1}, \gamma_{1}-1}(t-s) f(v(s, x)) d s,
\end{aligned}
$$

where we have used the following formula

$$
\left(\frac{d}{d z}\right)^{(m)}\left[z^{\beta-1} E_{\alpha, \beta}\left(z^{\alpha}\right)\right]=z^{\beta-m-1} E_{\alpha, \beta-m}\left(z^{\alpha}\right), \quad \Re(\beta-m)>0, m=0,1, \ldots
$$

Applying $J_{0 \mid t}^{2-\gamma_{1}}$ to both sides of $(3.4)$, we obtain

$$
\begin{aligned}
J_{0 \mid t}^{2-\gamma_{1}}\left(u_{t}-u_{1}\right)= & J_{0 \mid t}^{2-\gamma_{1}}\left(\partial_{t} \widetilde{E}_{\gamma_{1}, 1}(t, x)\right) u_{0}(x)+J_{0 \mid t}^{2-\gamma_{1}}\left(\partial_{t}\left(t \widetilde{E}_{\gamma_{1}, 2}(t, \cdot)\right) u_{1}(x)-u_{1}(x)\right) \\
& +J_{0 \mid t}^{2-\gamma_{1}}\left(\int_{0}^{t}(t-s)^{\gamma_{1}-2} \widetilde{E}_{\gamma_{1}, \gamma_{1}-1}(t-s, \cdot) f(v(s, x))\right) d s .
\end{aligned}
$$

On the other hand, we have

$$
\begin{aligned}
& J_{0 \mid t}^{2-\gamma_{1}}\left(\int_{0}^{t}(t-s)^{\gamma_{1}-2} E_{\gamma_{1}, \gamma_{1}-1}\left(-|\xi|^{2}(s-\tau)^{\gamma_{1}}\right) \widehat{f}(s, \xi) d s\right) \\
= & \frac{1}{\Gamma\left(2-\gamma_{1}\right)} \int_{0}^{t}(t-s)^{1-\gamma_{1}} \int_{0}^{s}(s-\tau)^{\gamma_{1}-2} E_{\gamma_{1}, \gamma_{1}-1}\left(-|\xi|^{2}(s-\tau)^{\gamma_{1}}\right) \widehat{f}(\tau, \xi) d \tau d s \\
= & \sum_{k=0}^{+\infty} \frac{(-1)^{k}|\xi|^{2 k}}{\Gamma\left(2-\gamma_{1}\right) \Gamma\left(\gamma_{1} k+\gamma_{1}-1\right)} \int_{0}^{t}(t-s)^{1-\gamma_{1}} \int_{0}^{s}(s-\tau)^{\gamma_{1}-2+\gamma_{1} k} \widehat{f}(\tau, \xi) d \tau d s \\
= & \sum_{k=0}^{+\infty} \frac{(-1)^{k}|\xi|^{2 k}}{\Gamma\left(2-\gamma_{1}\right) \Gamma\left(\gamma_{1} k+\gamma_{1}-1\right)} \int_{0}^{t} \int_{\tau}^{t}(t-s)^{1-\gamma_{1}}(s-\tau)^{\gamma_{1}-2+\gamma_{1} k} d s \widehat{f}(\tau, \xi) d \tau \\
= & \sum_{k=0}^{+\infty} \frac{(-1)^{k}|\xi|^{2 k}}{\Gamma\left(2-\gamma_{1}\right) \Gamma\left(\gamma_{1} k+\gamma_{1}-1\right)} \mathbf{B}\left(2-\gamma_{1}, \gamma_{1} k+\gamma_{1}-1\right) \int_{0}^{t}(t-s)^{\gamma_{1} k} \widehat{f}(s, \xi) d s \\
= & \int_{0}^{t} E_{\gamma_{1}, 1}\left(-|\xi|^{2}(s-\tau)^{\gamma_{1}}\right) \widehat{f}(s, \xi) d s .
\end{aligned}
$$


Here $B$ denotes to the beta function.

Applying the Fourier inverse transform to both sides of 3.5 yields

$$
J_{0 \mid t}^{2-\gamma_{1}}\left(\int_{0}^{t}(t-s)^{\gamma_{1}-2} \widetilde{E}_{\gamma_{1}, \gamma_{1}-1}(t-s, \cdot) f(v(s, x))\right) d s=\int_{0}^{t} \widetilde{E}_{\gamma_{1}, 1}(t-s, \cdot) f(v(s, x)) d s .
$$

Then, for every test function $\varphi \in C_{x, t}^{2,1}\left(\mathbb{R}^{N} \times[0, T]\right), \operatorname{supp} \varphi \subset \subset R^{N} \times[0, T]$ and $\varphi(T, x)=0$, we have

$$
\begin{aligned}
\int_{\mathbb{R}^{N}} J_{0 \mid t}^{2-\gamma_{1}}\left(u_{t}-u_{1}\right) \varphi d x= & \int_{\mathbb{R}^{N}} J_{0 \mid t}^{2-\gamma_{1}}\left(\partial_{t} \widetilde{E}_{\gamma_{1}, 1}(t, x)\right) u_{0}(x) \varphi d x \\
& +\int_{\mathbb{R}^{N}} J_{0 \mid t}^{2-\gamma_{1}}\left(\partial_{t}\left(t \widetilde{E}_{\gamma_{1}, 2}(t, \cdot)\right) u_{1}(x)-u_{1}(x)\right) \varphi d x \\
& +\int_{\mathbb{R}^{N}} \int_{0}^{t} \widetilde{E}_{\gamma_{1}, 1}(t-s) f(v(s, x)) d s \varphi d x
\end{aligned}
$$

Setting

$$
I:=\int_{\mathbb{R}^{N}} J_{0 \mid t}^{2-\gamma_{1}}\left(u_{t}-u_{1}\right) \varphi d x
$$

we get

$$
\begin{aligned}
\frac{\partial}{\partial t} I= & \int_{\mathbb{R}^{N}} \frac{\partial}{\partial t}\left[J_{0 \mid t}^{2-\gamma_{1}}\left(u_{t}-u_{1}\right) \varphi\right] d x \\
= & \int_{\mathbb{R}^{N}} \frac{\partial}{\partial t}\left[J_{0 \mid t}^{2-\gamma_{1}}\left(\partial_{t} \widetilde{E}_{\gamma_{1}, 1}(t, x)\right) u_{0}(x) \varphi\right] d x \\
& +\int_{\mathbb{R}^{N}} \frac{\partial}{\partial t}\left[J_{0 \mid t}^{2-\gamma_{1}}\left(\partial_{t}\left(t \widetilde{E}_{\gamma_{1}, 2}(t, \cdot)\right) u_{1}(x)-u_{1}(x)\right) \varphi\right] d x \\
& +\int_{\mathbb{R}^{N}} \frac{\partial}{\partial t}\left(\int_{0}^{t} \widetilde{E}_{\gamma_{1}, 1}(t-s) d \tau f(s, x) d s \varphi\right) d x
\end{aligned}
$$

On the other hand, using the relations

$$
\begin{aligned}
D_{0 \mid t}^{\gamma_{1}} \widetilde{E}_{\gamma_{1}, 1}(t, \cdot) u_{0}(x) & =\Delta \widetilde{E}_{\gamma_{1}, 1}(t, \cdot) u_{0}(x), \\
D_{0 \mid t}^{\gamma_{1}}\left(t \widetilde{E}_{\gamma_{1}, 2}(t, \cdot)\right) u_{1}(x) & =\Delta\left(t \widetilde{E}_{\gamma_{1}, 2}(t, \cdot)\right) u_{1}(x),
\end{aligned}
$$

we obtain

$$
\begin{aligned}
& \int_{\mathbb{R}^{N}} \frac{\partial}{\partial t}\left[J_{0 \mid t}^{2-\gamma_{1}}\left(\partial_{t}\left(t \widetilde{E}_{\gamma_{1}, 2}(t, \cdot)\right) u_{1}(x)-u_{1}(x)\right) \varphi\right] d x \\
= & \int_{\mathbb{R}^{N}} D_{0 \mid t}^{\gamma_{1}}\left(t \widetilde{E}_{\gamma_{1}, 2}(t, \cdot)\right) u_{1}(x) \varphi(t, x) d x \\
& +\int_{\mathbb{R}^{N}} J_{0 \mid t}^{2-\gamma_{1}}\left(\partial_{t}\left(t \widetilde{E}_{\gamma_{1}, 2}(t, \cdot)\right) u_{1}(x)-u_{1}(x)\right) \varphi_{t}(t, x) d x \\
= & \int_{\mathbb{R}^{N}} t \widetilde{E}_{\gamma_{1}, 2}(t, \cdot) u_{1}(x) \Delta \varphi(t, x) d x \\
& +\int_{\mathbb{R}^{N}} J_{0 \mid t}^{2-\gamma_{1}}\left(\partial_{t}\left(t \widetilde{E}_{\gamma_{1}, 2}(t, \cdot)\right) u_{1}(x)-u_{1}(x)\right) \varphi_{t}(t, x) d x,
\end{aligned}
$$


and

$$
\begin{aligned}
\int_{\mathbb{R}^{N}} \frac{\partial}{\partial t}\left[J_{0 \mid t}^{2-\gamma_{1}}\left(\partial_{t} \widetilde{E}_{\gamma_{1}, 1}(t, x)\right) u_{0}(x) \varphi\right] d x= & \int_{\mathbb{R}^{N}} \widetilde{E}_{\gamma_{1}, 1}(t, x) u_{0}(x) \Delta \varphi(t, x) d x \\
& +\int_{\mathbb{R}^{N}} J_{0 \mid t}^{2-\gamma_{1}}\left(\partial_{t} \widetilde{E}_{\gamma_{1}, 1}(t, x)\right) u_{0}(x) \varphi_{t}(t, x) d x .
\end{aligned}
$$

Using the Leibniz formula, we get

$$
\begin{aligned}
\frac{\partial}{\partial t} \int_{0}^{t} \widetilde{E}_{\gamma_{1}, 1}(t-s) f(v(s, x)) d s & =\widetilde{E}_{\gamma_{1}, 1}(0) f(v(t, x))+\int_{0}^{t} \partial_{t} \widetilde{E}_{\gamma_{1}, 1}(t-s) f(v(s, x)) d s \\
& =f(v(t, x))+\int_{0}^{t} \partial_{t} \widetilde{E}_{\gamma_{1}, 1}(t-s) f(v(t, x)) d s .
\end{aligned}
$$

So

$$
\begin{aligned}
\frac{\partial}{\partial t} I= & \int_{\mathbb{R}^{N}} \widetilde{E}_{\gamma_{1}, 1}(t, x) u_{0}(x) \Delta \varphi d x+\int_{\mathbb{R}^{N}} t \widetilde{E}_{\gamma_{1}, 2}(t, \cdot) u_{1}(x) \Delta \varphi d x \\
& +\int_{\mathbb{R}^{N}} f(v(t, x)) \varphi d x+\int_{\mathbb{R}^{N}} \int_{0}^{t}(t-s)^{\gamma_{1}-1} \widetilde{E}_{\gamma_{1}, \gamma_{1}}(t-s) f(v(s, x)) \Delta \varphi d s d x \\
& +\int_{\mathbb{R}^{N}} J_{0 \mid t}^{2-\gamma_{1}}\left(\partial_{t} \widetilde{E}_{\gamma_{1}, 1}(t, x)\right) u_{0}(x) \varphi_{t} d x \\
& +\int_{\mathbb{R}^{N}} J_{0 \mid t}^{2-\gamma_{1}}\left(\partial_{t}\left(t \widetilde{E}_{\gamma_{1}, 2}(t, \cdot)\right) u_{1}(x)-u_{1}(x)\right) \varphi_{t} d x \\
& +\int_{\mathbb{R}^{N}} \int_{0}^{t} \widetilde{E}_{\gamma_{1}, 1}(t-s) f(v(s, x)) d s \varphi_{t} d x .
\end{aligned}
$$

Using the fact that $u$ is a mild solution, we obtain

$$
\begin{aligned}
\frac{\partial}{\partial t} I= & \int_{\mathbb{R}^{N}} u \Delta \varphi d x+\int_{\mathbb{R}^{N}} f(v(t, x)) \varphi d x+\int_{\mathbb{R}^{N}} J_{0 \mid t}^{2-\gamma_{1}}\left(\partial_{t} \widetilde{E}_{\gamma_{1}, 1}(t, x)\right) u_{0}(x) \varphi_{t} d x \\
& +\int_{\mathbb{R}^{N}} J_{0 \mid t}^{2-\gamma_{1}}\left(\partial_{t}\left(t \widetilde{E}_{\gamma_{1}, 2}(t, \cdot)\right) u_{1}(x)-u_{1}(x)\right) \varphi_{t} d x \\
& +\int_{\mathbb{R}^{N}} \int_{0}^{t} \widetilde{E}_{\gamma_{1}, 1}(t-s) f(v(s, x)) d s \varphi_{t} d x \\
= & \int_{\mathbb{R}^{N}} u \Delta \varphi d x+\int_{\mathbb{R}^{N}} f(v(t, x)) \varphi d x+\int_{\mathbb{R}^{N}} J_{0 \mid t}^{2-\gamma_{1}}\left(u_{t}-u_{1}\right) \varphi_{t} d x
\end{aligned}
$$

On the other hand, we have

$$
\frac{\partial}{\partial t} I=\int_{\mathbb{R}^{N}} \frac{\partial}{\partial t}\left[J_{0 \mid t}^{2-\gamma_{1}}\left(u_{t}-u_{1}\right)\right] \varphi d x+\int_{\mathbb{R}^{N}} J_{0 \mid t}^{2-\gamma_{1}}\left(u_{t}-u_{1}\right) \varphi_{t} d x .
$$

Integrating both sides of $(3.6)$ and $(3.7)$ on $[0, T]$, and then identifying the terms, we get

$$
\int_{0}^{T} \int_{\mathbb{R}^{N}} \frac{\partial}{\partial t} J_{0 \mid t}^{2-\gamma_{1}}\left(u_{t}-u_{1}\right) \varphi d x d t=\int_{0}^{T} \int_{\mathbb{R}^{N}} u \Delta \varphi d x d t+\int_{0}^{T} \int_{\mathbb{R}^{N}} f(v(t, x)) \varphi d x d t .
$$


The formula of integration by parts allows to write

$$
\int_{0}^{T} \int_{\mathbb{R}^{N}}\left(u_{t}-u_{1}\right) D_{t \mid T}^{\gamma_{1}} \varphi d x d t=\int_{0}^{T} \int_{\mathbb{R}^{N}} u \Delta \varphi d x d t+\int_{0}^{T} \int_{\mathbb{R}^{N}} f(v(t, x)) \varphi d x d t
$$

By an analogous calculation, we can show that

$$
\int_{0}^{T} \int_{\mathbb{R}^{N}}\left(v_{t}-v_{1}\right) D_{t \mid T}^{\gamma_{2}} \varphi d x d t=\int_{0}^{T} \int_{\mathbb{R}^{N}} v \Delta \varphi d x d t+\int_{0}^{T} \int_{\mathbb{R}^{N}} g(u(t, x)) \varphi d x d t .
$$

This completes the proof.

Our next result concerns the blow-up of solutions of 1.1 .

Theorem 3.6 (Blow-up of mild solution). Let $N \geq 1, p>1, q>1, u_{0}, v_{0}, u_{1}, v_{1} \in$ $L_{\text {loc }}^{p}\left(\mathbb{R}^{N}\right), 1<\gamma_{1}, \gamma_{2}<2$, be such that $\int_{\mathbb{R}^{N}} u_{0}(x) d x \geq 0, \int_{\mathbb{R}^{N}} v_{0}(x) d x \geq 0, \int_{\mathbb{R}^{N}} u_{1}(x) d x>$ 0 and $\int_{\mathbb{R}^{N}} v_{1}(x) d x>0$. If

$$
\frac{N}{2}<\min \left\{\frac{1}{\gamma_{1}}+\frac{\gamma_{2} p+\gamma_{1}}{\gamma_{1}(p q-1)}, \frac{1}{\gamma_{1}}+\frac{p+1}{p q-1}\right\},
$$

or

$$
\frac{N}{2}<\min \left\{\frac{1-\gamma_{2}}{\gamma_{1}}+\frac{\left(p \gamma_{2}+\gamma_{1}\right) q}{\gamma_{1}(p q-1)}, \frac{1-\gamma_{2}}{\gamma_{1}}+\frac{(p+1) q}{p q-1}\right\}
$$

or

$$
\frac{N}{2}<\min \left\{\frac{\left(1-\gamma_{1}\right)}{\gamma_{2}}+\frac{\left(\gamma_{1} q+\gamma_{2}\right) p}{\gamma_{2}(p q-1)}, \frac{1-\gamma_{1}}{\gamma_{2}}+\frac{(q+1) p}{p q-1}\right\}
$$

or

$$
\frac{N}{2}<\min \left\{\frac{1}{\gamma_{2}}+\frac{q \gamma_{1}+\gamma_{2}}{(p q-1) \gamma_{2}}, \frac{1}{\gamma_{2}}+\frac{q+1}{p q-1}\right\},
$$

then the mild solution $(u, v)$ of $1.1-(1.2)$ blows up in a finite time.

\section{Global existence and decay estimates}

Proof of Theorem 3.3. The proof proceeds in three steps. Without loss of generality, we assume that $1<\gamma_{1} \leq \gamma_{2}<2$ and $q \geq p \geq 1$ such that $p q>1$.

First step: Global existence for $(u, v)$ in $L^{s_{1}}\left(\mathbb{R}^{N}\right) \times L^{s_{2}}\left(\mathbb{R}^{N}\right)$.

Since $p q>1$, from 3.3 we have for $N \geq 2$ that

$$
\frac{N}{2} \geq \max \left\{\frac{1}{\gamma_{1}}+\frac{q+1}{p q-1}, \frac{1}{\gamma_{1}}+\frac{p \gamma_{2}+\gamma_{1}}{\gamma_{1}(p q-1)}\right\} .
$$

If $\max \left\{\frac{1}{\gamma_{1}}+\frac{q+1}{p q-1}, \frac{1}{\gamma_{1}}+\frac{p \gamma_{2}+\gamma_{1}}{\gamma_{1}(p q-1)}\right\}=\frac{1}{\gamma_{1}}+\frac{q+1}{p q-1}$, then $\frac{N}{2} \geq \frac{1}{\gamma_{1}}+\frac{q+1}{p q-1}$, which gives

$$
1-\frac{p q-1}{q(p+1) \gamma_{2}}<1-\frac{p q-1}{2 q(p+1)}<\frac{p q-1+q \gamma_{1}+\gamma_{1}}{\gamma_{1} q(p+1)} \leq \frac{N(p q-1)}{2 q(p+1)} .
$$


If $\max \left\{\frac{1}{2}+\frac{q+1}{p q-1}, \frac{1}{\gamma_{1}}+\frac{p \gamma_{2}+\gamma_{1}}{\gamma_{1}(p q-1)}\right\}=\frac{1}{\gamma_{1}}+\frac{p \gamma_{2}+\gamma_{1}}{\gamma_{1}(p q-1)}$. That is $\frac{1}{\gamma_{1}}+\frac{q+1}{p q-1} \leq \frac{1}{\gamma_{1}}+\frac{p \gamma_{2}+\gamma_{1}}{\gamma_{1}(p q-1)}$, in this case

$$
\frac{N}{2} \geq \frac{1}{\gamma_{1}}+\frac{p \gamma_{2}+\gamma_{1}}{\gamma_{1}(p q-1)} \geq \frac{1}{\gamma_{1}}+\frac{q+1}{p q-1}
$$

which gives again $\frac{N(p q-1)}{2 q(p+1)}>1-\frac{p q-1}{q(p+1) \gamma_{2}}$, and since $1-\frac{p q-1}{q(p+1) \gamma_{2}}<1$, we can choose $\delta>0$ such that

$$
1-\frac{p q-1}{q(p+1) \gamma_{2}}<\delta<\min \left\{1, \frac{N(p q-1)}{2 q(p+1)}\right\} .
$$

We set

$$
\begin{aligned}
r_{1}=\frac{N \gamma_{1}(p q-1)}{2\left[\gamma_{1}(1+\delta p)+\gamma_{2} p(1-\delta)\right]}, & r_{2} & =\frac{N \gamma_{2}(p q-1)}{2\left[\gamma_{2}(1+\delta q)+\gamma_{1} q(1-\delta)\right]} \\
\frac{1}{s_{1}}=\frac{2 \delta}{N} \frac{p+1}{p q-1}, & \frac{1}{s_{2}} & =\frac{2 \delta}{N} \frac{q+1}{p q-1} \\
\sigma_{1}=\frac{(1-\delta)\left(\gamma_{1}+\gamma_{2} p\right)}{p q-1}, & \sigma_{2} & =\frac{(1-\delta)\left(\gamma_{2}+\gamma_{1} q\right)}{p q-1}
\end{aligned}
$$

Clearly, we have

$$
\begin{aligned}
& \frac{1}{r_{1}}=\frac{2}{N \gamma_{1}} \frac{(1-\delta)\left(\gamma_{1}+\gamma_{2} p\right)}{p q-1}+\frac{2 \delta}{N} \frac{(p+1)}{p q-1} \\
& \frac{1}{r_{2}}=\frac{2}{N \gamma_{2}} \frac{(1-\delta)\left(\gamma_{2}+\gamma_{1} q\right)}{p q-1}+\frac{2 \delta}{N} \frac{(q+1)}{p q-1}
\end{aligned}
$$

The choice of $\delta$ gives

$$
\delta>1-\frac{p q-1}{\left(\gamma_{2}+\gamma_{1} q\right) p} \quad \Longrightarrow \quad p \sigma_{2}=\frac{(1-\delta)\left(\gamma_{2}+\gamma_{1} q\right)}{p q-1} p<1
$$

and

$$
\delta>1-\frac{p q-1}{\left(\gamma_{1}+\gamma_{2} p\right) q} \quad \Longrightarrow \quad q \sigma_{1}=\frac{(1-\delta)\left(\gamma_{1}+\gamma_{2} p\right)}{p q-1} q<1
$$

It is easy to check that

$$
\begin{gathered}
s_{1}>q, \quad s_{2}>p, \quad p s_{1}>s_{2}, \quad q s_{2}>s_{1}, \quad s_{1}>r_{1}>1, \quad s_{2}>r_{2}>1 \\
\frac{N}{2} \gamma_{1}\left(\frac{1}{r_{1}}-\frac{1}{s_{1}}\right) q<1, \quad \frac{N}{2} \gamma_{2}\left(\frac{1}{r_{2}}-\frac{1}{s_{2}}\right) p<1
\end{gathered}
$$

and

$$
\frac{N}{2}\left(\frac{p}{s_{2}}-\frac{1}{s_{1}}\right)=\delta=\frac{N}{2}\left(\frac{q}{s_{1}}-\frac{1}{s_{2}}\right)
$$

One can easily verify that

$$
\delta>\frac{p q\left(\gamma_{1}-1\right)+1+p \gamma_{2}}{\left[\gamma_{1} q+\gamma_{2}\right] p} \Longleftrightarrow\left(\gamma_{2}-\frac{N}{2} \gamma_{2}\left(\frac{q}{s_{1}}-\frac{1}{s_{2}}\right)-q \sigma_{1}\right) p>-1
$$


Let $\left(u_{0}, v_{0}\right) \in L^{r_{1}}\left(\mathbb{R}^{N}\right) \times L^{r_{2}}\left(\mathbb{R}^{N}\right)$. Let $u \in C\left(\left[0, T_{\max }\right) ; L^{s_{1}}\left(\mathbb{R}^{N}\right)\right)$ and $v \in C\left(\left[0, T_{\max }\right) ;\right.$ $\left.L^{s_{2}}\left(\mathbb{R}^{N}\right)\right)$. For $t \in\left[0, T_{\max }\right)$, from $(1.1)$, we have

$$
\begin{aligned}
\|u(t, \cdot)\|_{s_{1}} \leq & \left\|\widetilde{E}_{\gamma_{1}, 1}(t) u_{0}\right\|_{s_{1}}+\left\|t \widetilde{E}_{\gamma_{1}, 2}(t, \cdot)\right\|_{s_{1}} \\
& +\int_{0}^{t}(t-\tau)^{\gamma_{1}-1}\left\|\widetilde{E}_{\gamma_{1}, \gamma_{1}}(t-\tau)|v(\tau, \cdot)|^{p}\right\|_{s_{1}} d \tau, \\
\|v(t, \cdot)\|_{s_{2}} \leq & \left\|\widetilde{E}_{\gamma_{2}, 1}(t) v_{0}\right\|_{s_{2}}+\left\|t \widetilde{E}_{\gamma_{2}, 2}(t, \cdot)\right\|_{s_{2}} \\
& +\int_{0}^{t}(t-\tau)^{\gamma_{2}-1}\left\|\widetilde{E}_{\gamma_{2}, \gamma_{2}}(t-\tau)|u(\tau, \cdot)|^{q}\right\|_{s_{2}} d \tau .
\end{aligned}
$$

Applying Lemmas 2.1 and 2.2, we get

$$
\begin{aligned}
\|u(t, \cdot)\|_{s_{1}} \leq & t^{-\sigma_{1}}\left\|u_{0}\right\|_{r_{1}}+t^{-\sigma_{1}}\left\|u_{1}\right\|_{\dot{\mathcal{H}}_{r_{1}}^{-\frac{2}{\gamma_{1}}}} \\
& +C \int_{0}^{t}(t-\tau)^{\gamma_{1}-1}(t-\tau)^{-\frac{N}{2} \gamma_{1}\left(\frac{p}{s_{2}}-\frac{1}{s_{1}}\right)}\|v(\tau, \cdot)\|_{s_{2}}^{p} d \tau, \\
\|v(t, \cdot)\|_{s_{2}} \leq & t^{-\sigma_{2}}\left\|v_{0}\right\|_{r_{2}}+t^{-\sigma_{1}}\left\|v_{1}\right\|_{\dot{\mathcal{H}}_{r_{2}}^{-\frac{2}{\gamma_{2}}}} \\
& +C \int_{0}^{t}(t-\tau)^{\gamma_{2}-1}(t-\tau)^{-\frac{N}{2} \gamma_{2}\left(\frac{q}{s_{1}}-\frac{1}{s_{2}}\right)}\|u(\tau, \cdot)\|_{s_{1}}^{q} d \tau .
\end{aligned}
$$

Using (4.5) into 4.4), we obtain

$$
\begin{aligned}
& \|u(t, \cdot)\|_{s_{1}} \\
\leq & \left(\left\|u_{0}\right\|_{r_{1}}+\left\|u_{1}\right\|_{\dot{\mathcal{H}}_{r_{1}}^{-\frac{2}{\gamma_{1}}}}\right) t^{-\sigma_{1}}+C \int_{0}^{t}(t-\tau)^{\gamma_{1}-1}(t-\tau)^{-\frac{N}{2} \gamma_{1}\left(\frac{p}{s_{2}}-\frac{1}{s_{1}}\right)} d \tau \\
& \times\left(\left(\left\|v_{0}\right\|_{r_{2}}+\left\|v_{1}\right\|_{\dot{\mathcal{H}}_{r_{2}}^{-\frac{2}{\gamma_{2}}}}\right) t^{-\sigma_{2}}+C \int_{0}^{t}(t-\tau)^{\gamma_{2}-1}(t-\tau)^{-\frac{N}{2} \gamma_{2}\left(\frac{q}{s_{1}}-\frac{1}{s_{2}}\right)}\|u(t, \cdot)\|_{s_{1}}^{q} d \tau\right)^{p},
\end{aligned}
$$

provided that $1-\frac{1}{\gamma_{1}}<\frac{N}{2}\left(\frac{q}{s_{1}}-\frac{1}{s_{2}}\right)<1$ and $1-\frac{1}{\gamma_{2}}<\frac{N}{2}\left(\frac{p}{s_{2}}-\frac{1}{s_{1}}\right)<1$ which are indeed satisfied.

Hence

$$
\begin{aligned}
& \|u(t, \cdot)\|_{s_{1}} \\
\leq & \left(\left\|u_{0}\right\|_{r_{1}}+\left\|u_{1}\right\|_{\dot{\mathcal{H}}_{r_{1}}-\frac{2}{\gamma_{1}}}\right) t^{-\sigma_{1}} \\
& +C \int_{0}^{t}(t-\tau)^{\gamma_{1}-1-\frac{N}{2} \gamma_{1}\left(\frac{p}{s_{2}}-\frac{1}{s_{1}}\right)} \tau^{-p \sigma_{2}} d \tau\left(\left\|v_{0}\right\|_{r_{2}}+\left\|v_{1}\right\|_{\dot{\mathcal{H}}_{r_{2}}^{-\frac{2}{\gamma_{2}}}}\right)^{p} \\
& +C \int_{0}^{t}(t-\tau)^{\gamma_{1}-1-\frac{N}{2} \gamma_{1}\left(\frac{p}{s_{2}}-\frac{1}{s_{1}}\right)} \tau \tau^{\left(\gamma_{2}-\frac{N}{2} \gamma_{2}\left(\frac{q}{s_{1}}-\frac{1}{s_{2}}\right)-q \sigma_{1}\right) p}\left(\tau^{\sigma_{1}}\|u(\tau, \cdot)\|_{s_{1}}\right)^{p q} d \tau .
\end{aligned}
$$


Multiplying both sides of 4.6 by $t^{\sigma_{1}}$ with $\sigma_{1}=\frac{(1-\delta)\left(\gamma_{1}+\gamma_{2} p\right)}{p q-1}$, we get

$$
\begin{aligned}
& t^{\sigma_{1}}\|u(t, \cdot)\|_{s_{1}} \\
\leq & \left\|u_{0}\right\|_{r_{1}}+\left\|u_{1}\right\|_{\dot{\mathcal{H}}_{r_{1}}^{-\frac{2}{\gamma_{1}}}} \\
& +C t^{\sigma_{1}} \int_{0}^{t}(t-\tau)^{\gamma_{1}-1-\frac{N}{2} \gamma_{1}\left(\frac{p}{s_{2}}-\frac{1}{s_{1}}\right)} \tau^{-p \sigma_{2}} d \tau\left(\left\|v_{0}\right\|_{r_{2}}+\left\|v_{1}\right\|_{\dot{\mathcal{H}}_{r_{2}}^{-\frac{2}{\gamma_{2}}}}\right)^{p} \\
& +C t^{\sigma_{1}} \int_{0}^{t}(t-\tau)^{\gamma_{1}-1-\frac{N}{2} \gamma_{1}\left(\frac{p}{s_{2}}-\frac{1}{s_{1}}\right)} \tau_{\tau}\left(\gamma_{2}-\frac{N}{2} \gamma_{2}\left(\frac{q}{s_{1}}-\frac{1}{s_{2}}\right)-q \sigma_{1}\right) p \\
& \left(\tau^{\sigma_{1}}\|u(\tau, \cdot)\|_{s_{1}}\right)^{p q} d \tau .
\end{aligned}
$$

Since $\gamma_{1}-1-\frac{N}{2} \gamma_{1}\left(\frac{p}{s_{2}}-\frac{1}{s_{1}}\right)>-1,\left(\gamma_{2}-\frac{N}{2} \gamma_{2}\left(\frac{q}{s_{1}}-\frac{1}{s_{2}}\right)-q \sigma_{1}\right) p>-1$, we have

$$
\begin{aligned}
t^{\sigma_{1}}\|u(t, \cdot)\|_{s_{1}} \leq & \left\|u_{0}\right\|_{r_{1}}+\left\|u_{1}\right\|_{\dot{\mathcal{H}}_{r_{1}}^{-\frac{2}{\gamma_{1}}}}+C t^{\sigma_{1}+\gamma_{1}-\frac{N}{2} \gamma_{1}\left(\frac{p}{s_{2}}-\frac{1}{s_{1}}\right)-p \sigma_{2}}\left(\left\|v_{0}\right\|_{r_{2}}^{p}+\left\|v_{1}\right\|_{\dot{\mathcal{H}}_{\gamma_{2}}^{-\frac{2}{\gamma_{2}}}}^{p}\right) \\
& +C t^{\sigma_{1}+\gamma_{1}-\frac{N}{2} \gamma_{1}\left(\frac{p}{s_{2}}-\frac{1}{s_{1}}\right)+\left(\gamma_{2}-\frac{N}{2} \gamma_{2}\left(\frac{q}{s_{1}}-\frac{1}{s_{2}}\right)-q \sigma_{1}\right) p}\left(\sup _{0 \leq \tau \leq t} \tau^{\sigma_{1}}\|u(\tau, \cdot)\|_{s_{1}}\right)^{p q} .
\end{aligned}
$$

Note that

$$
\begin{gathered}
\sigma_{1}=\frac{N}{2} \gamma_{1}\left(\frac{1}{r_{1}}-\frac{1}{s_{1}}\right) \\
\sigma_{1}+\gamma_{1}-\frac{N}{2} \gamma_{1}\left(\frac{p}{s_{2}}-\frac{1}{s_{1}}\right)-p \sigma_{2}=0 \\
\sigma_{1}+\gamma_{1}-\frac{N}{2} \gamma_{1}\left(\frac{p}{s_{2}}-\frac{1}{s_{1}}\right)+\left(\gamma_{2}-\frac{N}{2} \gamma_{2}\left(\frac{q}{s_{1}}-\frac{1}{s_{2}}\right)-q \sigma_{1}\right) p=0 \\
\sigma_{1}+\gamma_{1}-\gamma_{1} \delta+\left(\gamma_{2}-\gamma_{2} \delta-q \sigma_{1}\right) p=0
\end{gathered}
$$

Define $f(t)=\sup _{0 \leq \tau \leq t} \tau^{\sigma_{1}}\|u(\tau, \cdot)\|_{s_{1}}, t \in\left[0, T_{\max }\right)$. So we deduce from 4.7) that

$$
f(t) \leq C\left(\left\|u_{0}\right\|_{r_{1}}+\left\|u_{1}\right\|_{\dot{\mathcal{H}}_{r_{1}}^{-\frac{2}{\gamma_{1}}}}+\left\|v_{0}\right\|_{r_{2}}^{p}+\left\|v_{1}\right\|_{\dot{\mathcal{H}}_{r_{2}}^{-\frac{2}{\gamma_{2}}}}^{p}+f(t)^{p q}\right)
$$

for all $t \in\left(0, T_{\max }\right)$. Setting

$$
A=\left\|u_{0}\right\|_{r_{1}}+\left\|u_{1}\right\|_{\dot{\mathcal{H}}_{r_{1}}^{-\frac{2}{\gamma_{1}}}}+\left\|v_{0}\right\|_{r_{2}}^{p}+\left\|v_{1}\right\|_{\dot{\mathcal{H}}_{r_{2}}^{-\frac{2}{\gamma_{2}}}}^{p} .
$$

Now if we take $A$ small enough such that $A<(2 C)^{\frac{p q}{1-p q}}$, then it follows by continuity argument that 4.8 implies

$$
f(t) \leq 2 C A \text { for all } t \in\left[0, T_{\max }\right) .
$$

Indeed, if 4.9 is not true. That is to say $f\left(t_{0}\right)>2 C A$ holds true for some $t_{0} \in\left(0, T_{\max }\right)$. By the intermediate value theorem since $f$ is continuous, non-decreasing and $f(0)=0$, there exists $t_{1} \in\left(0, t_{0}\right)$ such that $f\left(t_{1}\right)=2 C A$. From 4.8$)$, we get

$$
2 C A=f\left(t_{1}\right) \leq C\left(A+f\left(t_{1}\right)^{p q}\right),
$$


from which, it yields

$$
2 C A \leq C\left(A+(2 C A)^{p q}\right)
$$

which is equivalent to

$$
A \geq(2 C)^{\frac{p q}{1-p q}} .
$$

This is a contradiction. Therefore, it follows that

$$
f(t) \leq 2 C A \text { for any } t \in\left[0, T_{\max }\right)
$$

Thus

$$
t^{\sigma_{1}}\|u(t, \cdot)\|_{s_{1}} \leq C \quad \text { for any } t \in\left[0, T_{\max }\right) .
$$

Similarly, we obtain

$$
t^{\sigma_{2}}\|v(t, \cdot)\|_{s_{2}} \leq C \quad \text { for any } t \in\left[0, T_{\max }\right) .
$$

Now, from 4.2, 4.3 and Lemma 2.2, we can easily see that

$$
\|u(t, \cdot)\|_{\infty},\|v(t, \cdot)\|_{\infty} \leq C \text { for any } t \in[0,1] .
$$

On the other hand, since $s_{1}$ and $s_{2}$ satisfy

$$
\frac{(1-\delta)(p+1) s_{1}}{(p q-1) s_{2}} \gamma_{2}<1, \quad \frac{(1-\delta)(q+1) s_{2}}{(p q-1) s_{1}} \gamma_{2}<1
$$

it follows from 4.2, 4.3, Lemmas 2.1 and 2.2 that

$$
\begin{aligned}
\|u(t, \cdot)\|_{s_{1}} \leq & \left\|\widetilde{E}_{\gamma_{1}, 1}(t) u_{0}\right\|_{s_{1}}+t\left\|\widetilde{E}_{\gamma_{2}, 2}(t) u_{1}\right\|_{s_{1}} \\
& +\int_{0}^{t}(t-\tau)^{\gamma_{1}-1}\left\|\widetilde{E}_{\gamma_{1}, \gamma_{1}}(t-\tau)|v(\tau, \cdot)|^{p}\right\|_{s_{1}} d \tau \\
\leq & C\left\|u_{0}\right\|_{s_{1}}+t\left\|u_{1}\right\|_{s_{1}}+C \int_{0}^{t}(t-\tau)^{\gamma_{1}-1}\left\||v(\tau, \cdot)|^{p}\right\|_{s_{1}} d \tau \\
\leq & C\left\|u_{0}\right\|_{s_{1}}+\left\|u_{1}\right\|_{s_{1}}+C \sup _{\tau \in(0, t)}\|v(\tau)\|_{\infty}^{p-\frac{s_{2}}{s_{1}}} \int_{0}^{t}(t-\tau)^{\gamma_{1}-1}\|v(\tau, \cdot)\|_{s_{2}}^{\frac{s_{2}}{s_{1}}} d \tau \\
\leq & C\left\|u_{0}\right\|_{s_{1}}+\left\|u_{1}\right\|_{s_{1}}+C \sup _{\tau \in(0, t)}\|v(\tau)\|_{\infty}^{p-\frac{s_{2}}{s_{1}}} \int_{0}^{t}\|v(\tau, \cdot)\|_{s_{2}}^{\frac{s_{2}}{s_{1}}} d \tau
\end{aligned}
$$

for all $t \in[0,1]$. Hence $\|u(t, \cdot)\|_{s_{1}} \leq C$ for any $t \in[0,1]$. Analogously,

$$
\|v(t, \cdot)\|_{s_{2}} \leq C \quad \text { for all } t \in[0,1] .
$$

From 4.10, 4.11, 4.13, 4.14 and Lemma 2.3, we conclude that

$$
\left\{\begin{array}{l}
\|u(t, \cdot)\|_{s_{1}} \leq C(t+1)^{-\frac{(1-\delta)\left(\gamma_{1}+p \gamma_{2}\right)}{p q-1}}, \\
\|u(t, \cdot)\|_{s_{2}} \leq C(t+1)^{-\frac{(1-\delta)\left(\gamma_{2}+q \gamma_{1}\right)}{p q-1}}
\end{array}\right.
$$


for all $t \in\left[0, T_{\max }\right)$.

Second step: $L^{\infty}$-global existence estimates of $(u, v)$ in $L^{\infty}\left(\mathbb{R}^{N}\right) \times L^{\infty}\left(\mathbb{R}^{N}\right)$.

Let $s_{1}, s_{2}$ be as in (4.1). Since $p \leq q$, we have

$$
\frac{N p}{2 s_{2}} \leq \frac{N q}{2 s_{1}}
$$

We further assume, for some $\xi>q$ and $w>p$, that $u(t) \in L^{w}\left(\mathbb{R}^{N}\right), v(t) \in L^{\xi}\left(\mathbb{R}^{N}\right)$, and

$$
\begin{cases}\|u(t, \cdot)\|_{w} \leq C\left(1+t^{k_{1}}\right), & t \in\left[0, T_{\max }\right), \\ \|v(t, \cdot)\|_{\xi} \leq C\left(1+t^{k_{2}}\right), & t \in\left[0, T_{\max }\right)\end{cases}
$$

holds true for some positive constants $k_{1}$ and $k_{2}$. Then, by (4.2), (4.3) and Lemma 2.2 , we have

$$
\begin{aligned}
& \|u(t, \cdot)\|_{\infty} \leq\left\|\widetilde{E}_{\gamma_{1}, 1}(t) u_{0}\right\|_{\infty}+t\left\|\widetilde{E}_{\gamma_{2}, 2}(t) u_{1}\right\|_{\infty}+\int_{0}^{t}(t-\tau)^{\gamma_{1}-1-\frac{N \gamma_{1} p}{2 \xi}}\|v(\tau, \cdot)\|_{\xi}^{p} d \tau \\
& \|v(t, \cdot)\|_{\infty} \leq\left\|\widetilde{E}_{\gamma_{2}, 1}(t) v_{0}\right\|_{\infty}+t\left\|\widetilde{E}_{\gamma_{2}, 2}(t) u_{1}\right\|_{\infty}+\int_{0}^{t}(t-\tau)^{\gamma_{2}-1-\frac{N \gamma_{2} q}{2 w}}\|u(\tau, \cdot)\|_{w}^{q} d \tau
\end{aligned}
$$

for all $t \in\left[0, T_{\max }\right)$. If one can find $\xi$ and $w$ such that

$$
\frac{N p}{2 \xi}<1 \quad \text { or } \quad \frac{N q}{2 w}<1
$$

then the $L^{\infty}$-estimates of $(u, v)$ is obtained. In fact, if $\frac{N p}{2 \xi}<1$, in view of 4.16$)$, it yields from 4.17) that

$$
\begin{aligned}
\|u(t, \cdot)\|_{\infty} & \leq\left\|\widetilde{E}_{\gamma_{1}, 1}(t) u_{0}\right\|_{\infty}+C \max _{\tau \in[0, t]}\|v(\tau, \cdot)\|_{\xi}^{p} t^{\left(1-\frac{N p}{2 \xi}\right) \gamma_{1}} \\
& \leq C\left(1+t^{\left(1-\frac{N p}{2 \xi}\right) \gamma_{1}+p k_{2}}\right)
\end{aligned}
$$

and by taking $w=\infty$ in 4.18 , we get

$$
\begin{aligned}
\|v(t, \cdot)\|_{\infty} \leq & \left\|\widetilde{E}_{\gamma_{2}, 1}(t) v_{0}\right\|_{\infty}+t\left\|\widetilde{E}_{\gamma_{2}, 2}(t) u_{1}\right\|_{\infty}+\int_{0}^{t}(t-\tau)^{\gamma_{2}-1}\|u(\tau, \cdot)\|_{\infty}^{q} d \tau \\
\leq & \left\|\widetilde{E}_{\gamma_{2}, 1}(t) v_{0}\right\|_{\infty}+t\left\|\widetilde{E}_{\gamma_{2}, 2}(t) u_{1}\right\|_{\infty} \\
& +\int_{0}^{t}(t-\tau)^{\gamma_{2}-1}\left(1+t^{\left(1-\frac{N p}{2 \xi}\right) \gamma_{1}+p k_{2}}\right)^{q} d \tau \\
\leq & C\left(1+t^{\gamma_{2}+\left[\left(1-\frac{N p}{2 \xi}\right) \gamma_{1}+p k_{2}\right] q}\right) .
\end{aligned}
$$

These estimates show that $T_{\max }=\infty$, and

$$
u, v \in L_{\mathrm{loc}}^{\infty}\left([0, \infty) ; L^{\infty}\left(\mathbb{R}^{N}\right)\right) .
$$


In a similar manner, we can establish the case $\frac{N q}{2 w}<1$. To find appropriate $\xi$ and $w$, we note that 4.20 and 4.21 hold by taking $\xi=s_{1}$ or $w=s_{2}$ if $\frac{N q}{2 s_{1}}<1$ or $\frac{N p}{2 s_{2}}<1$; this is certainly the case when $N \leq 2$ with $s_{1}>q$ and $s_{2}>p$.

Thus it remains to deal with the case $N>2, \frac{N q}{2 s_{1}} \geq 1$ and $\frac{N p}{2 s_{2}} \geq 1$. We do this via an iterative process. Define $s_{1}^{\prime}=s_{1}, s_{1}^{\prime \prime}=s_{2}$, since $s_{1}^{\prime}>q$ and $s_{1}^{\prime \prime}>p$, using the Hölder inequality and Lemmas 2.1 and 2.2 , we get from $(4.2),(4.3)$ that

$$
\begin{aligned}
& \|u(t, \cdot)\|_{s_{2}^{\prime}} \leq\left\|\widetilde{E}_{\gamma_{1}, 1}(t) u_{0}\right\|_{s_{2}^{\prime}}+t\left\|\widetilde{E}_{\gamma_{1}, 2}(t) u_{1}\right\|_{s_{2}^{\prime}}+\int_{0}^{t}(t-\tau)^{\gamma_{1}-1-\frac{N \gamma_{1}}{2}\left(\frac{p}{s_{2}^{\prime \prime}}-\frac{1}{s_{2}^{\prime}}\right)}\|v(\tau, \cdot)\|_{s_{2}^{\prime \prime}}^{p} d \tau, \\
& \|v(t, \cdot)\|_{s_{2}^{\prime \prime}} \leq\left\|\widetilde{E}_{\gamma_{2}, 1}(t) v_{0}\right\|_{s_{2}^{\prime \prime}}+t\left\|\widetilde{E}_{\gamma_{2}, 2}(t) u_{1}\right\|_{s_{2}^{\prime \prime}}+\int_{0}^{t}(t-\tau)^{\gamma_{2}-1-\frac{N \gamma_{2}}{2}\left(\frac{q}{s_{1}^{\prime}}-\frac{1}{s_{2}^{\prime \prime}}\right)}\|u(\tau, \cdot)\|_{s_{1}^{\prime}}^{q} d \tau,
\end{aligned}
$$

where $s_{2}^{\prime}$ and $s_{2}^{\prime \prime}$ are such that

$$
\frac{N}{2}\left(\frac{p}{s_{1}^{\prime \prime}}-\frac{1}{s_{2}^{\prime}}\right)<1, \quad \frac{N}{2}\left(\frac{q}{s_{1}^{\prime}}-\frac{1}{s_{2}^{\prime \prime}}\right)<1 .
$$

This can be shown by taking

$$
\frac{1}{s_{2}^{\prime}}=\frac{p}{s_{1}^{\prime \prime}}-\frac{2}{N}+\eta, \quad \frac{1}{s_{2}^{\prime \prime}}=\frac{q}{s_{1}^{\prime}}-\frac{2}{N}+\eta,
$$

where $0<\eta<\frac{2(1-\delta)}{N}$ with $\delta>1-\frac{1}{\gamma_{1}}$. Namely

$$
\frac{N}{2}\left(\frac{p}{s_{1}^{\prime \prime}}-\frac{1}{s_{2}^{\prime}}\right)=\frac{N}{2}\left(\frac{q}{s_{1}^{\prime}}-\frac{1}{s_{2}^{\prime \prime}}\right)=1-\frac{N}{2} \eta>1-\frac{1}{\gamma_{1}} .
$$

Observe that, since $\delta>1-\frac{p q-1}{q(p+1) \gamma_{2}}>1-\frac{1}{\gamma_{2}}$, we have

$$
\begin{aligned}
1-\frac{1}{\gamma_{1}}<\frac{N}{2}\left(\frac{p}{s_{1}^{\prime \prime}}-\frac{1}{s_{2}^{\prime}}\right)<1, & 1-\frac{1}{\gamma_{2}}<\frac{N}{2}\left(\frac{q}{s_{1}^{\prime}}-\frac{1}{s_{2}^{\prime \prime}}\right)<1, \\
\frac{1}{s_{1}^{\prime}}-\frac{1}{s_{2}^{\prime}}=\frac{2}{N}(1-\delta)-\eta>0, & \frac{1}{s_{1}^{\prime \prime}}-\frac{1}{s_{2}^{\prime \prime}}=\frac{2}{N}(1-\delta)-\eta>0,
\end{aligned}
$$

and hence $s_{2}^{\prime}>s_{1}^{\prime}>q$ and $s_{2}^{\prime \prime}>s_{1}^{\prime \prime}>p$.

Next, define the sequences $\left\{s_{i}^{\prime}\right\}_{i \geq 1}$ and $\left\{s_{i}^{\prime \prime}\right\}_{i \geq 1}$, iteratively, as follows

$$
\frac{1}{s_{i}^{\prime}}=\frac{p}{s_{i-1}^{\prime \prime}}-\frac{2}{N}+\eta, \quad \frac{1}{s_{i}^{\prime \prime}}=\frac{q}{s_{i-1}^{\prime}}-\frac{2}{N}+\eta, \quad i \geq 3 .
$$

Then

$$
\begin{aligned}
& \frac{1}{s_{i}^{\prime}}-\frac{1}{s_{i+1}^{\prime}}=p\left(\frac{1}{s_{i-1}^{\prime \prime}}-\frac{1}{s_{i}^{\prime \prime}}\right)=p q\left(\frac{1}{s_{i-2}^{\prime}}-\frac{1}{s_{i-1}^{\prime}}\right), \\
& \frac{1}{s_{i}^{\prime \prime}}-\frac{1}{s_{i+1}^{\prime \prime}}=q\left(\frac{1}{s_{i-1}^{\prime}}-\frac{1}{s_{i}^{\prime}}\right)=p q\left(\frac{1}{s_{i-2}^{\prime \prime}}-\frac{1}{s_{i-1}^{\prime \prime}}\right) .
\end{aligned}
$$


Since $p q>1$, in view of 4.23 , we get

$$
\frac{1}{s_{i}^{\prime}}>\frac{1}{s_{i+1}^{\prime}}, \quad \frac{1}{s_{i}^{\prime \prime}}>\frac{1}{s_{i+1}^{\prime \prime}}, \quad i \geq 1,
$$

and

$$
\lim _{i \rightarrow+\infty}\left(\frac{1}{s_{i}^{\prime}}-\frac{1}{s_{i+1}^{\prime}}\right)=\lim _{i \rightarrow+\infty}\left(\frac{1}{s_{i}^{\prime \prime}}-\frac{1}{s_{i+1}^{\prime \prime}}\right)=+\infty .
$$

Now, we ensure that there exists $i_{0}$ such that

$$
\frac{p}{s_{i_{0}}^{\prime \prime}}<\frac{2}{N} \quad \text { or } \quad \frac{q}{s_{i_{0}}^{\prime}}<\frac{2}{N}
$$

On the contrary, that is, $\frac{p}{s_{i}^{\prime \prime}} \geq \frac{2}{N}$ and $\frac{q}{s_{i}^{\prime}} \geq \frac{2}{N}$ for all $i \geq 1$. Then, by 4.24, we see that $s_{i}^{\prime}>0, s_{i}^{\prime \prime}>0$ for all $i \geq 1$ and hence, by 4.25,

$$
q<s_{1}^{\prime}<\cdots<s_{i}^{\prime}<\cdots, \quad p<s_{1}^{\prime \prime}<\cdots<s_{i}^{\prime \prime}<\cdots
$$

which contradicts 4.26.

Let $i_{0}$ be the smallest number satisfying (4.27). Notice that $i_{0} \geq 2$. Without loss of generality, we assume that

$$
\frac{p}{s_{i_{0}}^{\prime \prime}}<\frac{2}{N}, \quad \frac{p}{s_{i}^{\prime \prime}} \geq \frac{2}{N} \text { for any } 1 \leq i \leq i_{0}-1, \quad \frac{q}{s_{i}^{\prime}} \geq \frac{2}{N} \text { for any } 1 \leq i \leq i_{0} .
$$

It then follows from 4.24 that

$$
s_{i}^{\prime}>0 \text { for any } 1 \leq i \leq i_{0}, \quad s_{i}^{\prime \prime}>0 \text { for any } 1 \leq i \leq i_{0}+1,
$$

which together with 4.25 leads to

$$
q<\cdots<s_{i_{0}-1}^{\prime}<s_{i_{0}}^{\prime}, \quad p<\cdots<s_{i_{0}}^{\prime \prime}<s_{i_{0}+1}^{\prime \prime} .
$$

Now, from 4.24, we have, for all $i \geq 2$,

$$
\frac{N}{2}\left(\frac{p}{s_{i-1}^{\prime \prime}}-\frac{1}{s_{i}^{\prime}}\right)=1-\frac{N}{2} \eta=\frac{N}{2}\left(\frac{q}{s_{i-1}^{\prime}}-\frac{1}{s_{i}^{\prime \prime}}\right) .
$$

Now, let us deal with the boundedness of $(u(t, \cdot), v(t, \cdot))$ in $L^{s_{i}^{\prime}}\left(\mathbb{R}^{N}\right) \times L^{s_{i}^{\prime \prime}}\left(\mathbb{R}^{N}\right)$. Using the Hölder inequality, Lemmas 2.1 and 2.2, it follows from $3.1-(3.2)$, inductively, that

$$
\begin{aligned}
\|u(t, \cdot)\|_{s_{i}^{\prime} \leq} & \left\|\widetilde{E}_{\gamma_{1}, 1}(t) u_{0}\right\|_{s_{i}^{\prime}}+t\left\|\widetilde{E}_{\gamma_{2}, 2}(t) u_{1}\right\|_{s_{i}^{\prime}} \\
& +C \int_{0}^{t}(t-\tau)^{\gamma_{1}-1-\frac{N}{2} \gamma_{1}\left(\frac{p}{s_{i-1}^{\prime \prime}}-\frac{1}{s_{i}^{\prime}}\right)}\|v(\tau, \cdot)\|_{s_{i-1}^{\prime \prime}}^{p} d \tau \\
\leq & C\left\|u_{0}\right\|_{s_{i}^{\prime}}+t\left\|u_{1}\right\|_{s_{i}^{\prime}}+C \int_{0}^{t}(t-\tau)^{\gamma_{1}-1-\gamma_{1}}\left(1-\frac{N}{2} \eta\right)\|v(\tau, \cdot)\|_{s_{i-1}^{\prime \prime}}^{p} d \tau
\end{aligned}
$$


for any $2 \leq i \leq i_{0}, t \in\left(0, T_{\max }\right)$ and

$$
\begin{aligned}
\|v(t, \cdot)\|_{s_{i}^{\prime \prime} \leq} & \left\|\widetilde{E}_{\gamma_{2}, 1}(t) v_{0}\right\|_{s_{i}^{\prime \prime}}+t\left\|\widetilde{E}_{\gamma_{2}, 2}(t) v_{1}\right\|_{s_{i}^{\prime \prime}} \\
& +C \int_{0}^{t}(t-\tau)^{\gamma_{2}-1+\frac{N}{2} \gamma_{2}\left(\frac{q}{s_{i-1}^{\prime}}-\frac{1}{s_{i}^{\prime \prime}}\right)}\|u(\tau, \cdot)\|_{s_{i-1}^{\prime}}^{q} d \tau \\
\leq & C\left\|v_{0}\right\|_{s_{i}^{\prime \prime}}+C\left\|v_{1}\right\|_{s_{i}^{\prime \prime}}+C \int_{0}^{t}(t-\tau)^{\gamma_{2}-1-\gamma_{2}}\left(1-\frac{N \eta}{2}\right)\|u(\tau, \cdot)\|_{s_{i-1}^{\prime}}^{q} d \tau
\end{aligned}
$$

for any $t \in\left(0, T_{\max }\right)$ and for any $2 \leq i \leq i_{0}+1$.

It clearly follows from 4.29 and 4.30 that $u(t) \in L^{s_{i}^{\prime}}\left(\mathbb{R}^{N}\right), v(t) \in L^{s_{i}^{\prime \prime}}\left(\mathbb{R}^{N}\right)$ :

$$
\left\{\begin{array}{lll}
u(t, \cdot) \in L^{s_{i}^{\prime}}\left(\mathbb{R}^{N}\right), & \|u(t, \cdot)\|_{s_{i}^{\prime}} \leq C\left(1+t^{a_{i}}\right), & 1 \leq \forall i \leq i_{0}, t \in\left(0, T_{\max }\right), \\
v(t, \cdot) \in L^{s_{i}^{\prime \prime}}\left(\mathbb{R}^{N}\right), & \|v(t, \cdot)\|_{s_{i}^{\prime \prime}} \leq C\left(1+t^{b_{i}}\right), & 1 \leq \forall i \leq i_{0}+1, t \in\left(0, T_{\max }\right)
\end{array}\right.
$$

for some positive constants $a_{i}, b_{i}$. Since $\frac{N p}{2 s_{i_{0}^{\prime \prime}}}<1$, taking $s_{2}=s_{i_{0}}^{\prime \prime}$, 4.19) holds. In consequence, we get $T_{\max }=+\infty$ and that 4.22 holds.

Third step: $L^{\infty}$-decay estimates.

Let

$$
\sigma_{1}=\frac{(1-\delta)\left(p \gamma_{2}+\gamma_{1}\right)}{(p q-1)}, \quad \sigma_{2}=\frac{(1-\delta)\left(q \gamma_{1}+\gamma_{2}\right)}{(p q-1)} .
$$

If $\frac{p N}{2 s_{2}}<1$, by taking $\xi=s_{2}$ in 4.18) and using 4.15, we get

$$
\begin{aligned}
\|u(t, \cdot)\|_{\infty} \leq & C t^{-\frac{N \gamma_{1}}{2 r_{1}}}\left\|u_{0}\right\|_{r_{1}}+C t^{1-\frac{N \gamma_{1}}{2 m}}\left\|u_{1}\right\|_{m} \\
& +C \int_{0}^{t}(t-\tau)^{\gamma_{1}-1-\frac{N \gamma_{1}}{2} \frac{p}{s_{2}}} \tau^{-p \sigma_{2}} d \tau .
\end{aligned}
$$

From 3.3 with $p q>2 q+3$, we get $\frac{N}{2 r_{1}}<1$ and for any $m$ depending on $N$ such that $\frac{N}{2}<m<\frac{N \gamma_{1}}{2}, N \geq 2$, we infer that

$$
1-\frac{N \gamma_{1}}{2 m}<0 \text { and } \frac{N}{2 m}<1
$$

On the other hand, since

$$
p \sigma_{2}<1, \quad \gamma_{1}-\frac{N \gamma_{1}}{2} \frac{p}{s_{2}}-p \sigma_{2}=-\frac{\left[\gamma_{1}+\gamma_{1} p \delta+(1-\delta) p \gamma_{2}\right]}{p q-1}
$$

and

$$
\frac{\gamma_{1}+\gamma_{1} p \delta+p \gamma_{2}(1-\delta)}{p q-1}=\frac{N \gamma_{1}}{2 r_{1}}
$$

it follows from 4.32 and 4.33 that

$$
\|u(t, \cdot)\|_{\infty} \leq C t^{-\frac{N}{2 r_{1}} \gamma_{1}}+C t^{1-\frac{N}{2 m} \gamma_{1}}+C t^{-\frac{\left[\gamma_{1}+\gamma_{1} p \delta+(1-\delta) p \gamma_{2}\right]}{p q-1}} .
$$


Therefore, we have from 4.31, 4.34) and Lemma 2.3 that

$$
\|u(t, \cdot)\|_{\infty} \leq C(1+t)^{-\min \left\{\frac{N}{2 r_{1}} \gamma_{1}, \frac{N}{2 m} \gamma_{1}-1\right\}} \quad \text { for any } t \geq 0 .
$$

Similarly, for $\frac{q N}{2 s_{1}}<1$ we find that

$$
\|v(t, \cdot)\|_{\infty} \leq C(1+t)^{-\min \left\{\frac{N}{2 r_{2}} \gamma_{2}, 1-\frac{N}{2 m} \gamma_{2}\right\}} \quad \text { for any } t \geq 0 .
$$

Also, 4.34) holds as $p N /\left(2 s_{2}\right) \leq q N /\left(2 s_{1}\right)$.

In particular, if $p q>\gamma_{2}(q+1)+1$, we can choose $\delta>1-\frac{p q-1}{q(p+1) \gamma_{2}}$ and $\delta \approx 1-\frac{p q-1}{q(p+1) \gamma_{2}}$ such that $q N /\left(2 s_{1}\right)<1$. Therefore, the estimates (4.34) and (4.35) hold. It is useful to note that $N \leq 2$ implies $q N /\left(2 s_{1}\right)<1$ and $q N /\left(2 s_{1}\right)<1$ implies $p q>\gamma_{2}(q+1)+1$.

It remains to consider the following two cases:

$\triangleright N>2, \frac{N p}{2 s_{2}}<1$ and $\frac{N q}{2 s_{1}} \geq 1$.

Let

$$
\sigma^{\prime}=\frac{\gamma_{1}+\gamma_{1} p \delta+(1-\delta) p \gamma_{2}}{p q-1}
$$

For positive $\mu$ such that $\mu<\min \left\{\sigma^{\prime}, \sigma_{1}\right\}$ and $q \mu<1$; Since $N>2$ and $q>1$, we can choose $k>0$ such that $k>\frac{q N}{2}$ and $q \mu+\frac{q N \gamma_{2}}{2 k}>\gamma_{2}$. Since $s_{1} \leq q N / 2$, we have $k>s_{1}$. By the interpolation inequality,

$$
\|u(t)\|_{k} \leq\|u(t)\|_{\infty}^{\left(k-s_{1}\right) / k}\|u(t)\|_{s_{1}}^{s_{1} / k} \leq C t^{-\sigma^{\prime}\left(k-s_{1}\right) / k} t^{-\sigma_{1} s_{1} / k} \quad \text { for any } t>0 .
$$

Therefore, by 4.10, 4.34, we have

$$
\|u(t)\|_{k} \leq C t^{-\mu} \text { for all } t>0 .
$$

Consequently, for any $t>0$,

$$
\begin{aligned}
\|v(t)\|_{\infty} & \leq\left\|\widetilde{E}_{\gamma_{2}, 1}(t) v_{0}\right\|_{\infty}+t\left\|\widetilde{E}_{\gamma_{2}, 2}(t) v_{1}\right\|_{\infty}+C \int_{0}^{t}(t-\tau)^{\gamma_{2}-1-\frac{N q}{2 k} \gamma_{2}}\|u(\tau)\|_{k}^{q} d \tau \\
& \leq C t^{-\frac{N}{2 r_{2}} \gamma_{2}}\left\|v_{0}\right\|_{r_{2}}+C t^{1-\frac{N}{2 r_{2}} \gamma_{2}}\left\|v_{1}\right\|_{r_{2}}+C \int_{0}^{t}(t-\tau)^{\gamma_{2}-1-\frac{N \gamma_{2} q}{2 k}} \tau^{-q \mu} d \tau \\
& \leq C\left(t^{-\frac{N}{2} \gamma_{2}}+t^{1-\frac{N}{2 r_{2}} \gamma_{2}}+t^{\gamma_{2}-\frac{N \gamma_{2} q}{2 k}-q \mu}\right) \\
& \leq C t^{-\alpha}
\end{aligned}
$$

where $\alpha=\min \left\{\frac{N}{2 r_{2}} \gamma_{2}-1,-\gamma_{2}+\frac{N \gamma_{2} q}{2 k}+q \mu\right\}>0$,

$$
k>s_{1}, \quad q \mu<1, \quad k>q, \quad \gamma_{2}-\frac{N q \gamma_{2}}{2 k}>0, \quad \gamma_{2}-\frac{N q \gamma_{2}}{2 k}-q \mu<0 .
$$

From 4.12 and 4.36, we infer that

$$
\|v(t)\|_{\infty} \leq C(1+t)^{-\alpha} \text { for all } t \geq 0
$$


In case $p=1$ and $q^{2}>1+4 q$, we can choose $\delta>(1+3 q) /(p+1) q \gamma_{2}=(1+3 q) /\left(2 \gamma_{2} q\right)$ and $\delta \approx(1+3 q) /\left(2 \gamma_{2} q\right)$ such that $N /\left(2 s_{2}\right)<1$. Thus we obtain the estimate (4.34).

$\triangleright N>2, q N /\left(2 s_{1}\right) \geq 1, p N /\left(2 s_{2}\right) \geq 1, q \geq p>1$ and $\gamma_{1} \leq \gamma_{2}$.

This case needs a careful handling and we need to restrict further the choice of $\delta$. As $\sqrt{\frac{(p+1) q \gamma_{1}}{(q+1) p}}<\gamma_{1} \leq \gamma_{2}<2, p q>1$, it follows that $1-\frac{p q-1}{q(p+1) \gamma_{2}}<1-\frac{(p q-1)}{p(q+1) \gamma_{1}^{2}}$. We can select $\delta$ such that

$$
1-\frac{p q-1}{q(p+1) \gamma_{2}}<\delta<\min \left\{\frac{N(p q-1)}{2(p+1) q}, 1-\frac{p q-1}{p(q+1) \gamma_{1}^{2}}\right\} .
$$

Then we get immediately that $p \sigma_{2}>1 / \gamma_{1}>1 / q \gamma_{1}$ and $q \sigma_{1}>1 / \gamma_{1}>1 / p \gamma_{2}$.

Further, we notice that there exist $\varepsilon \in(0,1)$ and $\beta<1$ close to 1 such that

$$
p \sigma_{2}-\varepsilon>\frac{1}{\gamma_{1}}>\frac{1}{q \gamma_{1}}, \quad q \sigma_{1}-\varepsilon>\frac{1}{\gamma_{2}}>\frac{1}{p \gamma_{2}}, \quad \text { and } \quad \frac{1}{\gamma_{1}}<\beta-\varepsilon
$$

Letting $\eta=2 \varepsilon(1-\delta) / N$, we find the integer $i_{0}$ as in Step 2, and, without loss of generality, assume that (4.28) holds. We choose $\beta$ in addition to (4.37) satisfying

$$
\gamma_{1}<\gamma_{1} \frac{p N}{2 s_{i_{0}}^{\prime \prime}}+\beta, \quad \text { since } 1-\frac{1}{\gamma_{1}}<\frac{p N}{2 s_{i_{0}}^{\prime \prime}}
$$

As

$$
\delta<\frac{N(p q-1)}{2(p+1) q} \leq \frac{N(p q-1)}{2(q+1) p}, \quad \text { and } \quad \beta<1,
$$

we have

$$
\beta+\frac{(p+1) q \delta}{(p q-1)}<1+\frac{N}{2}, \quad \beta+\frac{(q+1) p \delta}{(p q-1)}<1+\frac{N}{2} .
$$

For $2 \leq i \leq i_{0}-1$, define $r_{i+1}^{\prime}$ and $r_{i+1}^{\prime \prime}$, inductively, as follows:

$$
\begin{aligned}
\frac{1}{r_{2}^{\prime}} & =\frac{1}{s_{2}^{\prime}}+\frac{2}{N}\left(p \sigma_{2}-\varepsilon(1-\delta)\right), & \frac{1}{r_{2}^{\prime \prime}} & =\frac{1}{s_{2}^{\prime \prime}}+\frac{2}{N}\left(q \sigma_{1}-\varepsilon(1-\delta)\right), \\
\frac{1}{r_{i+1}^{\prime}} & =\frac{1}{s_{i+1}^{\prime}}+\frac{2}{N}(\beta-\varepsilon(1-\delta)), & \frac{1}{r_{i+1}^{\prime \prime}} & =\frac{1}{s_{i+1}^{\prime \prime}}+\frac{2}{N}(\beta-\varepsilon(1-\delta)) .
\end{aligned}
$$

It is clear that $r_{i}^{\prime}, r_{i}^{\prime \prime}>0$ and $r_{i}^{\prime}<s_{i}^{\prime}, r_{i}^{\prime \prime}<s_{i}^{\prime \prime}$ for all $2 \leq i \leq i_{0}$. A simple calculation shows that $r_{i}^{\prime}, r_{i}^{\prime \prime}>1$.

As $s_{i}^{\prime}$ and $s_{i}^{\prime \prime}$ are increasing in $i$ for $1 \leq i \leq i_{0}$, we have

$$
\begin{aligned}
\frac{1}{r_{i+1}^{\prime}} & <\frac{1}{s_{2}^{\prime}}+\frac{2}{N}(\beta-\varepsilon(1-\delta)) \\
& =\frac{p}{s_{1}^{\prime \prime}}-\frac{2}{N}+\frac{2}{N} \varepsilon(1-\delta)+\frac{2}{N}(\beta-\varepsilon(1-\delta)) \\
& =\frac{2}{N}\left(\frac{p(q+1) \delta}{p q-1}+\beta-1\right)<1
\end{aligned}
$$


from 4.38, i.e., $r_{i+1}^{\prime}>1$.

Similarly, we can find that $r_{i+1}^{\prime \prime}>1$.

From 4.22 and 4.31 , we infer that there exists a positive constant $\widehat{C}$ such that, for any $0 \leq t \leq 1$,

$$
\|u(t)\|_{\infty},\|v(t)\|_{\infty},\|u(t)\|_{k_{1}},\|v(t)\|_{k_{2}} \leq \widehat{C}, \quad s_{1}^{\prime} \leq k_{1} \leq s_{i_{0}}^{\prime}, \quad s_{1}^{\prime \prime} \leq k_{2} \leq s_{i_{0}}^{\prime \prime} .
$$

Further, since $1-\eta N / 2=1-\varepsilon(1-\delta)$ and $p \sigma_{2}<1$, using 4.29, 4.30, 4.10) and 4.11, we arrive at the estimate

$$
\begin{aligned}
\|u(t, \cdot)\|_{s_{2}^{\prime}} \leq & \left\|\widetilde{E}_{\gamma_{1}, 1}(t) u_{0}\right\|_{s_{2}^{\prime}}+t\left\|\widetilde{E}_{\gamma_{1}, 2}(t) u_{1}\right\|_{s_{2}^{\prime}} \\
& +C \int_{0}^{t}(t-\tau)^{\gamma_{1}-1-\gamma_{1}(1-\varepsilon(1-\delta))}\|u(\tau, \cdot)\|_{s_{1}^{\prime \prime}}^{p} d \tau
\end{aligned}
$$

from which, we get

$$
\begin{aligned}
\|u(t, \cdot)\|_{s_{2}^{\prime}} \leq & C t^{-\frac{N}{2} \gamma_{1}\left(\frac{1}{r_{2}^{\prime}}-\frac{1}{s_{2}^{\prime}}\right)}\left\|u_{0}\right\|_{r_{2}^{\prime}}+t^{-\frac{N}{2} \gamma_{1}\left(\frac{1}{r_{2}^{\prime}}-\frac{1}{s_{2}^{\prime}}\right)}\left\|u_{1}\right\|_{\dot{\mathcal{H}}_{r_{2}^{\prime}}^{-\frac{2}{\gamma_{1}}}} \\
& +C \int_{0}^{t}(t-\tau)^{\gamma_{1}-1-\gamma_{1}(1-\varepsilon(1-\delta))} \tau^{-p \sigma_{2}} d \tau .
\end{aligned}
$$

Therefore

$$
\begin{aligned}
\|u(t, \cdot)\|_{s_{2}^{\prime}} \leq & C t^{-\gamma_{1}\left(p \sigma_{2}-\varepsilon(1-\delta)\right)}\left\|u_{0}\right\|_{r_{2}^{\prime}}+t^{-\gamma_{1}\left(p \sigma_{2}-\varepsilon(1-\delta)\right)}\left\|u_{1}\right\|_{\dot{\mathcal{H}}_{r_{2}^{\prime}}^{-\frac{2}{\gamma_{1}}}} \\
& +C \int_{0}^{t}(t-\tau)^{\gamma_{1}-1-\gamma_{1}(1-\varepsilon(1-\delta))} \tau^{-p \sigma_{2}} d \tau \\
\leq & C t^{-\gamma_{1}\left(p \sigma_{2}-\varepsilon(1-\delta)\right)} \quad \text { for any } t>0 .
\end{aligned}
$$

Similarly,

$$
\|v(t, \cdot)\|_{s_{2}^{\prime \prime}} \leq C t^{-\gamma_{2}\left(q \sigma_{1}-\varepsilon(1-\delta)\right)} \text { for any } t>0 .
$$

In view of 4.37) and $\beta<1$, thanks to Lemma 2.3, for any $t>0$, we conclude that

$$
\|u(t, \cdot)\|_{s_{2}^{\prime}} \leq C t^{-\gamma_{1} \beta / q} \text { and }\|v(t, \cdot)\|_{s_{2}^{\prime \prime}} \leq C t^{-\gamma_{2} \beta / p} .
$$

An iterative argument leads to

$$
\|u(t, \cdot)\|_{s_{i_{0}}^{\prime}} \leq C t^{-\gamma_{1}(\beta-\varepsilon(1-\delta))} \leq C t^{-\beta / q}, \quad\|v(t, \cdot)\|_{s_{i_{0}}^{\prime \prime}} \leq C t^{-\gamma_{2}(\beta-\varepsilon(1-\delta))} \leq C t^{-\beta / p}
$$

for any $t \geq 1$. Therefore, by 4.17) and 4.18, we have

$$
\begin{aligned}
\|u(t, \cdot)\|_{\infty} & \leq C t^{-\frac{N}{2 r_{1}} \gamma_{1}}\left\|u_{0}\right\|_{r_{1}}+C t^{1-\frac{N}{2 m} \gamma_{1}}\left\|u_{1}\right\|_{m}+C \int_{0}^{t}(t-\tau)^{\gamma_{1}-1-\gamma_{1} \frac{p N}{2 s_{i_{0}}^{\prime \prime}}}\|v(\tau, \cdot)\|_{s_{i_{0}}^{\prime \prime}}^{p} d \tau \\
& \leq C t^{-\frac{N}{2 r_{1}} \gamma_{1}}\left\|u_{0}\right\|_{r_{1}}+C t^{1-\frac{N}{2 m} \gamma_{1}}\left\|u_{1}\right\|_{m}+C \int_{0}^{t}(t-\tau)^{\gamma_{1}-1-\gamma_{1} \frac{p N}{2 s_{i_{0}}^{\prime \prime}}} \tau^{-\beta} d \tau .
\end{aligned}
$$


So

$$
\|u(t, \cdot)\|_{\infty} \leq C\left(t^{-\frac{N}{2 r_{1}} \gamma_{1}}+t^{1-\frac{N}{2 m} \gamma_{1}}+t^{\gamma_{1}-\gamma_{1} \frac{p N}{2 s_{i}^{\prime \prime}}-\beta}\right) \leq C t^{-\widetilde{\sigma}},
$$

where $\widetilde{\sigma}=\min \left\{\frac{N}{2 r_{1}} \gamma_{1}, \frac{N}{2 m} \gamma_{1}-1, \gamma_{1} \frac{p N}{2 s_{i_{0}}^{\prime \prime}}-\gamma_{1}+\beta\right\}>0$ from 4.39).

In view of the fact that $\frac{N q}{2 s_{1}} \geq 1$, we can make use of the arguments similar to the ones employed for the case $\frac{N p}{2 s_{2}}<1$ and $\frac{N q}{2 s_{1}} \geq 1$ to obtain $\|v(t, \cdot)\|_{\infty} \leq C t^{-\widehat{\sigma}}$ for some $\widehat{\sigma}>0$ and for every $t>0$. This completes the proof.

Remark 4.1. In the particular case: $N>2, q N /\left(2 s_{1}\right) \geq 1, p N /\left(2 s_{2}\right) \geq 1, q>p=1$ and $q \leq 3$, using the above method, we obtain

$$
\|u(t, \cdot)\|_{\infty} \leq C t^{-\widetilde{\sigma}} \text { for any } t>0
$$

where $\widetilde{\sigma}=\min \left\{\frac{N}{2} \gamma_{1}, \frac{N}{2 m} \gamma_{1}-1, \frac{p N}{2 s_{i_{0}}^{\prime \prime}} \gamma_{1}-\gamma_{1}+\gamma_{2}(\beta-\varepsilon(1-\delta))\right\}$. Here, $\varepsilon>0$ can be arbitrarily small, and $\beta$ can be arbitrarily close to 1 . However, since $s_{i_{0}}^{\prime \prime}$ depends on $\varepsilon$ and $s_{i_{0}}^{\prime \prime}$ is decreasing in $\varepsilon$, it is not clear that $\widetilde{\sigma}$ positive.

Proof of Theorem 3.6. The proof proceeds by contradiction. Suppose that $(u, v)$ is a mild solution of 1.1 which exists globally in time. Set

$$
\varphi(t, x)=\varphi_{1}(x) \varphi_{2}(t)
$$

where $\varphi_{1}(x)=\Phi^{l}\left(\frac{|x|}{T^{\lambda}}\right)$ with $\Phi \in C_{0}^{\infty}(\mathbb{R}), 0 \leq \Phi(z) \leq 1$, that satisfies

$$
\Phi(z)=\left\{\begin{array}{ll}
1 & \text { if }|z| \leq 1, \\
0 & \text { if }|z|>2
\end{array} \text { and } \quad \varphi_{2}(t)= \begin{cases}\left(1-\frac{t}{T}\right)^{l} & \text { if } t \leq T \\
0 & \text { if } t>T\end{cases}\right.
$$

where $l>\max \left\{1, \frac{q}{q-1} \gamma_{1}-1, \frac{p}{p-1} \gamma_{2}-1\right\}$ and $\lambda>0$ to be determined later.

We set $Q_{T}:=R^{N} \times[0, T]$. From Definition 3.4, we have

$$
\begin{aligned}
& \int_{Q_{T}} u D_{t \mid T}^{\gamma_{1}} \varphi(t, x) d x d t-\int_{Q_{T}} u \Delta \varphi(t, x) d x d t \\
= & \int_{\mathbb{R}^{N}} u_{0}(x)\left(D_{t \mid T}^{\gamma_{1}-1} \varphi\right)(0, \cdot) d x+\int_{Q_{T}} u_{1}(x) D_{t \mid T}^{\gamma_{1}-1} \varphi(t, x) d x d t+\int_{Q_{T}}|v(t, x)|^{p} \varphi(t, x) d x d t, \\
& \int_{Q_{T}} v D_{t \mid T}^{\gamma_{2}} \varphi(t, x) d x d t-\int_{Q_{T}} v \Delta \varphi(t, x) d x d t \\
= & \int_{\mathbb{R}^{N}} v_{0}(x)\left(D_{t \mid T}^{\gamma_{2}-1} \varphi\right)(0, \cdot) d x+\int_{Q_{T}} v_{1}(x) D_{t \mid T}^{\gamma_{2}-1} \varphi(t, x) d x d t+\int_{Q_{T}}|u(t, x)|^{q} \varphi(t, x) d x d t .
\end{aligned}
$$


On the other hand, we have from the definition of $\varphi$ that

$$
\begin{aligned}
& \int_{Q_{T}} u \varphi_{1}(x) D_{t \mid T}^{\gamma_{1}} \varphi_{2}(t) d x d t-\int_{Q_{T}} u \varphi_{2}(t) \Delta \varphi_{1}(x) d x d t \\
= & \int_{\mathbb{R}^{N}} u_{0}(x) \varphi_{1}(x)\left(D_{t \mid T}^{\gamma_{1}-1} \varphi_{2}\right)(0, \cdot) d x+\int_{Q_{T}} u_{1} \varphi_{1}(x) D_{t \mid T}^{\gamma_{1}-1} \varphi_{2}(t) d x d t \\
& +\int_{Q_{T}}|v(t, x)|^{p} \varphi_{1}(x) \varphi_{2}(t) d x d t
\end{aligned}
$$

and

$$
\begin{aligned}
& \int_{Q_{T}} v \varphi_{1}(x) D_{t \mid T}^{\gamma_{2}} \varphi_{2}(t) d x d t-\int_{Q_{T}} v \varphi_{2}(t) \Delta \varphi_{1}(x) d x d t \\
= & \int_{\mathbb{R}^{N}} v_{0}(x) \varphi_{1}(x)\left(D_{t \mid T}^{\gamma_{2}-1} \varphi_{2}\right)(0, \cdot) d x+\int_{Q_{T}} v_{1}(x) \varphi_{1}(x) D_{t \mid T}^{\gamma_{2}-1} \varphi_{2}(t) d x d t \\
& +\int_{Q_{T}}|u(t, x)|^{q} \varphi_{1}(x) \varphi_{2}(t) d x d t .
\end{aligned}
$$

Applying Hölder's inequality with exponents $q$ and $q^{\prime}=\frac{q}{q-1}$ to the right-hand side of (4.40, we get

$$
\begin{aligned}
\int_{Q_{T}} u \varphi_{1}(x) D_{t \mid T}^{\gamma_{1}} \varphi_{2}(t) d x d t & =\int_{Q_{T}} u\left|\varphi_{2}(t)\right|^{\frac{1}{q}}\left|\varphi_{1}(x)\right|^{1-\frac{1}{q}+\frac{1}{q}}\left|\varphi_{2}(t)\right|^{-\frac{1}{q}} D_{t \mid T^{\prime}}^{\gamma_{1}} \varphi_{2}(t) d x d t \\
& \leq \mathcal{I}^{\frac{1}{q}} \widetilde{\mathcal{A}}
\end{aligned}
$$

where we have set

$$
\begin{gathered}
\mathcal{I}:=\int_{Q_{T}}|u|^{q} \varphi_{1}(x) \varphi_{2} d x d t \\
\widetilde{\mathcal{A}}:=\left(\int_{Q_{T}}\left|D_{t \mid T}^{\gamma_{1}} \varphi_{2}(t)\right|^{q^{\prime}}\left|\varphi_{2}(t)\right|^{-\frac{q^{\prime}}{q}}\left|\varphi_{1}(x)\right|\left(1-\frac{1}{q}\right) q^{\prime} d x d t\right)^{\frac{1}{q^{\prime}}}, \\
\int_{Q_{T}} u \Delta \varphi_{1}(x) \varphi_{2}(t) d x d t \\
\leq \mathcal{I}^{\frac{1}{q}}\left(\int_{Q_{T}}\left|\Delta \varphi_{1}(x)\right|^{q^{\prime}}\left|\varphi_{1}(x)\right|^{-\frac{q^{\prime}}{q}}\left|\varphi_{2}(t)\right|^{\left(1-\frac{1}{q}\right) q^{\prime}} d x d t\right)^{\frac{1}{q^{\prime}}} \\
\leq C \mathcal{I}^{\frac{1}{q}}\left(\int_{\operatorname{supp}\left(\Delta \varphi_{1}\right)} \varphi_{1}^{1-q^{\prime}}(x)\left|\Delta \varphi_{1}(x)\right|^{q^{\prime}}\left|\varphi_{1}^{l}(x)\right|^{-\frac{q^{\prime}}{q}} d x \int_{0}^{T}\left|\varphi_{2}(t)\right|^{\left(1-\frac{1}{q}\right) q^{\prime}} d t\right)^{\frac{1}{q^{\prime}}} .
\end{gathered}
$$

Collecting the above estimates, we obtain

$$
\begin{aligned}
& C T^{\left(1-\gamma_{1}\right)} \int_{\mathbb{R}^{N}} u_{0}(x) \varphi_{1}(x) d x+C T^{2-\gamma_{1}} \int_{\mathbb{R}^{N}} u_{1}(x) \varphi_{1}(x) d x+\mathcal{J} \\
\leq & \mathcal{I}^{\frac{1}{q}} \widetilde{\mathcal{A}}+\mathcal{I}^{\frac{1}{q}}\left(\int_{\mathbb{R}^{N}}\left|\Delta \varphi_{1}(x)\right|^{q^{\prime}}\left|\varphi_{1}(x)\right|^{-\frac{q^{\prime}}{q}} d x \int_{0}^{T}\left|\varphi_{2}(t)\right|^{\left(1-\frac{1}{q}\right) q^{\prime}} d t\right)^{\frac{1}{q^{\prime}}}
\end{aligned}
$$


where we have set

$$
\mathcal{J}:=\int_{Q_{T}}|v|^{p} \varphi_{1}(x) \varphi_{2}(t) d x d t
$$

Similarly, we obtain

$$
\begin{aligned}
& \mathcal{I}+T^{\left(1-\gamma_{2}\right)} \int_{\mathbb{R}^{N}} v_{0} \varphi_{1}(x) d x+C T^{2-\gamma_{2}} \int_{\mathbb{R}^{N}} v_{1}(x) \varphi_{1}(x) d x \\
\leq & \mathcal{J}^{\frac{1}{p}}\left(\int_{Q_{T}}\left|D_{t \mid T^{\prime}}^{\gamma_{2}} \varphi_{2}(t)\right|^{p^{\prime}}\left|\varphi_{2}(t)\right|^{-\frac{p^{\prime}}{p}}\left|\varphi_{1}(x)\right|^{\left(1-\frac{1}{p}\right) p^{\prime}} d x d t\right)^{\frac{1}{p^{\prime}}} \\
& +\mathcal{J}^{\frac{1}{p}}\left(\int_{\mathbb{R}^{N}}\left|\Delta \varphi_{1}(x)\right|^{p^{\prime}}\left|\varphi_{1}(x)\right|^{-\frac{p^{\prime}}{p}} d x \int_{0}^{T}\left|\varphi_{2}(t)\right|\left(1-\frac{1}{p}\right) p^{\prime} d t\right)^{\frac{1}{p^{\prime}}},
\end{aligned}
$$

where $p p^{\prime}=p+p^{\prime}$. Consequently,

$$
\mathcal{J}+C T^{1-\gamma_{1}} \int_{\mathbb{R}^{N}} u_{0}(x) \varphi_{1}(x) d x+C T^{2-\gamma_{1}} \int_{\mathbb{R}^{N}} u_{1}(x) \varphi_{1}(x) d x \leq \mathcal{A} \mathcal{I}^{\frac{1}{q}},
$$

and

$$
\mathcal{I}+C T^{1-\gamma_{2}} \int_{\mathbb{R}^{N}} v_{0}(x) \varphi_{1}(x) d x+C T^{2-\gamma_{2}} \int_{\mathbb{R}^{N}} v_{1}(x) \varphi_{1}(x) d x \leq \mathcal{B} \mathcal{J}^{\frac{1}{p}}
$$

with

$$
\begin{aligned}
& \mathcal{A}=\left(\int_{Q_{T}}\left|D_{t \mid T}^{\gamma_{1}} \varphi_{2}(t)\right|^{q^{\prime}}\left|\varphi_{2}(t)\right|^{-\frac{q^{\prime}}{q}}\left|\varphi_{1}(x)\right|^{\left(1-\frac{1}{q}\right) q^{\prime}} d x d t\right)^{\frac{1}{q^{\prime}}} \\
& +\left(\int_{Q_{T}}\left|\Delta \varphi_{1}(x)\right|^{q^{\prime}}\left|\varphi_{1}(x)\right|^{-\frac{q^{\prime}}{q}}\left|\varphi_{2}(t)\right|^{\left(1-\frac{1}{q}\right) q^{\prime}} d x d t\right)^{\frac{1}{q^{\prime}}} \\
& \leq C T^{\left(-q^{\prime} \gamma_{1}+1+N \lambda\right) \frac{1}{q^{\prime}}}+C T^{\left(-2 \lambda q^{\prime}+1+N \lambda\right) \frac{1}{q^{\prime}}},
\end{aligned}
$$

and

$$
\begin{aligned}
& \mathcal{B}=\left(\int_{Q_{T}}\left|D_{t \mid T}^{\gamma_{2}} \varphi_{2}(t)\right|^{p^{\prime}}\left|\varphi_{2}(t)\right|^{-\frac{p^{\prime}}{p}}\left|\varphi_{1}(x)\right|\left(1-\frac{1}{p}\right) p^{\prime} d x d t\right)^{\frac{1}{p^{\prime}}} \\
& +\left(\int_{Q_{T}}\left|\Delta \varphi_{1}(x)\right|^{p^{\prime}}\left|\varphi_{1}^{l}(x)\right|^{-\frac{p^{\prime}}{p}}\left|\varphi_{2}(t)\right|^{\left(1-\frac{1}{p}\right) p^{\prime}} d x d t\right)^{\frac{1}{p^{\prime}}} \\
& \leq C T^{\left(-\gamma_{2} p^{\prime}+1+N \lambda\right) \frac{1}{p^{\prime}}}+C T^{\left(-2 \lambda p^{\prime}+1+N \lambda\right) \frac{1}{p^{\prime}}} \text {. }
\end{aligned}
$$

Using inequalities 4.41 and 4.42, we can write

$$
\mathcal{J}+C T^{2-\gamma_{1}} \int_{\mathbb{R}^{N}} u_{1}(x) \varphi_{1}(x) d x \leq \mathcal{A} \mathcal{B}^{\frac{1}{q}} \mathcal{J}^{\frac{1}{p q}}
$$

and

$$
\mathcal{I}+C T^{2-\gamma_{2}} \int_{\mathbb{R}^{N}} v_{1}(x) \varphi_{1}(x) d x \leq \mathcal{B} \mathcal{A}^{\frac{1}{p}} \mathcal{I}^{\frac{1}{p q}}
$$


Now, applying Young's inequality to the right hand side of the above estimates, we get

$$
(p q-1) \mathcal{J}+C p q T^{2-\gamma_{1}} \int_{\mathbb{R}^{N}} u_{1}(x) \varphi_{1}(x) d x \leq(p q-1)\left(\mathcal{A} \mathcal{B}^{\frac{1}{q}}\right)^{\frac{p q}{p q-1}},
$$

and

$$
(p q-1) \mathcal{I}+C p q T^{2-\gamma_{2}} \int_{\mathbb{R}^{N}} v_{1}(x) \varphi_{1}(x) d x \leq(p q-1)\left(\mathcal{B} \mathcal{A}^{\frac{1}{p}}\right)^{\frac{p q}{p q-1}} .
$$

At this stage, we set $x=T^{\lambda} y, t=T \tau$, with $\lambda>0$ to be chosen later. Then we have

$$
\begin{aligned}
\mathcal{A} \mathcal{B}^{\frac{1}{q}} \leq & C\left(T^{\left(-\gamma_{1} q^{\prime}+1+N \lambda\right) \frac{1}{q^{\prime}}}+T^{\left(-2 \lambda q^{\prime}+1+N \lambda\right) \frac{1}{q^{\prime}}}\right) \\
& \times\left(T^{\left(-\gamma_{2} p^{\prime}+1+N \lambda\right) \frac{1}{p^{\prime} q}}+T^{\left(-2 \lambda p^{\prime}+1+N \lambda\right) \frac{1}{p^{\prime} q}}\right)
\end{aligned}
$$

and

$$
\begin{aligned}
& \mathcal{B} \mathcal{A}^{\frac{1}{p}} \leq C\left(T^{\left(-\gamma_{2} p^{\prime}+1+N \lambda\right) \frac{1}{p^{\prime}}}+T^{\left(-2 \lambda p^{\prime}+1+N \lambda\right) \frac{1}{p^{\prime}}}\right) \\
& \times\left(T^{\left(-q^{\prime} \gamma_{1}+1+N \lambda\right) \frac{1}{q^{\prime}}}+T^{\left(-2 \lambda q^{\prime}+1+N \lambda\right) \frac{1}{q^{\prime}}}\right)^{\frac{1}{p}} .
\end{aligned}
$$

We choose $\lambda=\frac{\gamma_{1}}{2}$ so that $\left.\left(-q^{\prime} \gamma_{1}+1+N \lambda\right) \frac{1}{q^{\prime}}=\left(-2 \lambda q^{\prime}+1+N \lambda\right) \frac{1}{q^{\prime}}\right)$. Therefore, we have

$$
\int_{\mathbb{R}^{N}} u_{1}(x) \varphi_{1}(x) d x \leq C T^{\delta_{1}}
$$

and

$$
\int_{\mathbb{R}^{N}} v_{1}(x) \varphi_{1}(x) d x \leq C T^{\delta_{2}}
$$

where

$$
\begin{aligned}
\delta_{1}=\max \{ & {\left[\left(-q^{\prime} \gamma_{1}+1+\frac{\gamma_{1}}{2} N\right) \frac{1}{q^{\prime}}+\left(-p^{\prime} \gamma_{2}+1+N \frac{\gamma_{1}}{2}\right) \frac{1}{p^{\prime} q}\right] \frac{p q}{p q-1}+\gamma_{1}-2, } \\
& {\left.\left[\left(-q^{\prime} \gamma_{1}+1+\frac{\gamma_{1}}{2} N\right) \frac{1}{q^{\prime}}+\left(-p^{\prime} \gamma_{1}+1+N \frac{\gamma_{1}}{2}\right) \frac{1}{q p^{\prime}}\right] \frac{p q}{p q-1}+\gamma_{1}-2\right\}, }
\end{aligned}
$$

and

$$
\begin{aligned}
\delta_{2}=\max \{ & {\left[\left(-\gamma_{2} p^{\prime}+1+N \frac{\gamma_{1}}{2}\right) \frac{1}{p^{\prime}}+\left(-q^{\prime} \gamma_{1}+1+N \frac{\gamma_{1}}{2}\right) \frac{1}{p q^{\prime}}\right] \frac{p q}{p q-1}+\gamma_{2}-2, } \\
& {\left.\left[\left(-\gamma_{1} p^{\prime}+1+N \frac{\gamma_{1}}{2}\right) \frac{1}{p^{\prime}}+\left(-q^{\prime} \gamma_{1}+1+N \frac{\gamma_{1}}{2}\right) \frac{1}{p q^{\prime}}\right] \frac{p q}{p q-1}+\gamma_{2}-2\right\} . }
\end{aligned}
$$

The condition (3.3) leads to either $\delta_{1}<0$ or $\delta_{2}<0$. Then, as $T \rightarrow \infty$, the right-hand side of 4.43) (resp. (4.44) tends to zero and the left-hand side converges to $\int_{\mathbb{R}^{N}} u_{1}(x) d x>0$ (resp. $\int_{\mathbb{R}^{N}} v_{1}(x) d x>0$ ), which is contradiction.

We repeat the same argument with $\lambda=\frac{\gamma_{2}}{2}$ to conclude the proof of Theorem 3.6 
Remark 4.2. In the single equation case, when $\gamma_{1}=\gamma_{2}=\gamma$, we recover the case studied by [3]. In the system case, when $\gamma_{1}, \gamma_{2} \rightarrow 1$ with $\left(u_{1}, v_{1}\right) \equiv(0,0)$, we recover the classical Fujita exponent that studied by [13]. Moreover, letting $\gamma_{1}, \gamma_{2} \rightarrow 2$, we have shown in particular for $q \geq p>1$ and $N=3$ blow-up in finite time result holds for $\frac{3}{2}<\frac{q+1}{p q-1}$, which is subcritical exponent for the classical system of wave equations (see [8,9]).

\section{Acknowledgments}

This project was funded by the Deanship of Scientific Research (DSR), King Abdulaziz University, Jeddah, Saudi Arabia under grant no. (KEP-PHD-80-130-42). The authors, therefore, acknowledge with thanks DSR technical and financial support.

\section{References}

[1] M. F. de Almeida and L. C. F. Ferreira, Self-similarity, symmetries and asymptotic behavior in Morrey spaces for a fractional wave equation, Differential Integral Equations 25 (2012), no. 9-10, 957-976.

[2] M. F. de Almeida and J. C. P. Precioso, Existence and symmetries of solutions in Besov-Morrey spaces for a semilinear heat-wave type equation, J. Math. Anal. Appl. 432 (2015), no. 1, 338-355.

[3] M. F. de Almeida and A. Viana, Self-similar solutions for a superdiffusive heat equation with gradient nonlinearity, Electron. J. Differential Equations 250 (2016), 20 pp.

[4] C. Bandle, H. A. Levine and Q. S. Zhang, Critical exponents of Fujita type for inhomogeneous parabolic equations and systems, J. Math. Anal. Appl. 251 (2000), no. 2, 624-648.

[5] M. Berbiche, Existence and blow-up of solutions for damped wave system with nonlinear memory, Appl. Anal. 94 (2015), no. 12, 2535-2564.

[6] M. Berbiche and A. Hakem, Necessary conditions for the existence and sufficient conditions for the nonexistence of solutions to a certain fractional telegraph equation, Mem. Differ. Equ. Math. Phys. 56 (2012), 37-55.

[7] W. Chen and S. Holm, Modified Szabo's wave equation models for lossy media obeying frequency power law, J. Acoust. Soc. Am. 114 (2003), no. 5, 2570-2574.

[8] D. Del Santo and È. Mitidieri, Blow-up of solutions of a hyperbolic system: The critical case, Differential Equations 34 (1998), no. 9, 1157-1163. 
[9] K. Deng, Blow-up of solutions of some nonlinear hyperbolic systems, Rocky Mountain J. Math. 29 (1999), no. 3, 807-820.

[10] _ Nonexistence of global solutions of a nonlinear hyperbolic system, Trans. Amer. Math. Soc. 349 (1997), no. 4, 1685-1696.

[11] K. Deng and H. A. Levine, The role of critical exponents in blow-up theorems: The sequel, J. Math. Anal. Appl. 243 (2000), no. 1, 85-126.

[12] S. D. Eidelman and A. N. Kochubei, Cauchy problem for fractional diffusion equations, J. Differential Equations 199 (2004), no. 2, 211-255.

[13] M. Escobedo and M. A. Herrero, Boundedness and blow up for a semilinear reactiondiffusion system, J. Differential Equations 89 (1991), no. 1, 176-202.

[14] M. Fila, H. A. Levine and Y. Uda, A Fujita-type global existence-global non-existence theorem for a system of reaction diffusion equations with differing diffusivities, Math. Methods Appl. Sci. 17 (1994), no. 10, 807-835.

[15] A. Z. Fino and M. Kirane, Qualitative properties of solutions to a time-space fractional evolution equation, Quart. Appl. Math. 70 (2012), no. 1, 133-157.

[16] Y. Fujita, Cauchy problems of fractional order and stable processes, Japan J. Appl. Math. 7 (1990), no. 3, 459-476.

[17] A. Hakem and M. Berbiche, Blow up results for fractional differential equations and systems, Publ. Inst. Math. (Beograd) (N.S.) 93 (2013), no. 107, 173-186.

[18] H. Hirata and C. Miao, Space-time estimates of linear flow and application to some nonlinear integro-differential equations corresponding to fractional-order time derivative, Adv. Differential Equations 7 (2002), no. 2, 217-236.

[19] J. Kappler, S. Shrivastava, M. F. Schneider and R. R. Netz, Nonlinear fractional waves at elastic interfaces, Phys. Rev. Fluids 2 (2017), no. 11, 114804, 18 pp.

[20] J. Kemppainen, J. Siljander and R. Zacher, Representation of solutions and large-time behavior for fully nonlocal diffusion equations, J. Differential Equations 263 (2017), no. 1, 149-201.

[21] K.-H. Kim and S. Lim, Asymptotic behaviors of fundamental solution and its derivatives to fractional diffusion-wave equations, J. Korean Math. Soc. 53 (2016), no. 4, 929-967. 
[22] M. Kirane, Y. Laskri and N.-e. Tatar, Critical exponents of Fujita type for certain evolution equations and systems with spatio-temporal fractional derivatives, J. Math. Anal. Appl. 312 (2005), no. 2, 488-501.

[23] R. L. Magin, Fractional calculus models of complex dynamics in biological tissues, Comput. Math. Appl. 59 (2010), no. 5, 1586-1593.

[24] F. Mainardi, Fractional Calculus and Waves in Linear Viscoelasticity: An introduction to mathematical models, Imperial College Press, London, 2010.

[25] R. Metzler and J. Klafter, The random walk's guide to anomalous diffusion: A fractional dynamics approach, Phys. Rep. 339 (2000), no. 1, 77 pp.

[26] È. Mitidieri and S. I. Pokhozhaev, A priori estimates and the absence of solutions of nonlinear partial differential equations and inequalities, Proc. Steklov Inst. Math. 234 (2001), no. 3, 1-362.

[27] P. Y. H. Pang, F. Sun and M. Wang, Existence and non-existence of global solutions for a higher-order semilinear parabolic system, Indiana Univ. Math. J. 55 (2006), no. $3,1113-1134$.

[28] M. A. Pozio and A. Tesei, Global existence of solutions for a strongly coupled semilinear parabolic system, in: Recent Advances in Nonlinear Elliptic and Parabolic Problems (Nancy, 1988), 172-183, Pitman Res. Notes Math. Ser. 208, Longman Sci. Tech., Harlow, 1989.

[29] R. Redlinger, Pointwise a priori bounds for strongly coupled semilinear parabolic systems, Indiana Univ. Math. J. 36 (1987), no. 2, 441-454.

[30] A. A. Samarskii, V. A. Galaktionov, S. P. Kurdyumov and A. P. Mikhailov, Blowup in Quasilinear Parabolic Equations, Translated from the 1987 Russian original by Michael Grinfeld and revised by the authors, De Gruyter Expositions in Mathematics 19, Walter de Gruyter \& Co., Berlin, 1995.

[31] S. G. Samko, A. A. Kilbas and O. I. Marichev, Fractional Integrals and Derivatives: Theory and applications, Gordon and Breach Science Publishers, Yverdon, 1993.

[32] P. Straka, M. M. Meerschaert, R. J. McGough and Y. Zhou, Fractional wave equations with attenuation, Fract. Calc. Appl. Anal. 16 (2013), no. 1, 262-272.

[33] V. E. Tarasov, Fractional Dynamics: Applications of fractional calculus to dynamics of particles, fields and media, Nonlinear Physical Science, Springer, Heidelberg, 2010. 
[34] Q.-G. Zhang and H.-R. Sun, The blow-up and global existence of solutions of Cauchy problems for a time fractional diffusion equation, Topol. Methods Nonlinear Anal. 46 (2015), no. 1, 69-92.

Bashir Ahmad and Ahmed Alsaedi

Nonlinear Analysis and Applied Mathematics (NAAM) Research Group, Department of Mathematics, Faculty of Science, King Abdulaziz University, P.O. Box 80203, Jeddah 21589, Saudi Arabia

E-mail addresses: bashirahmad_qau@yahoo.com, aalsaedi@hotmail.com

Mohamed Berbiche

Biskra University, Laboratory of Mathématical Analysis, Probability and Optimizations, B.P. 145, 07000, Biskra, Algeria

E-mail addresses: mohamed.berbiche@univ-biskra.dz, berbichemed@yahoo.fr

\section{Mokhtar Kirane}

Department of Mathematics, College of Art and Sciences, Khalifa University of Science and Technology, Abu Dhabi, United Arab Emirates

and

Nonlinear Analysis and Applied Mathematics (NAAM) Research Group, Department of Mathematics, Faculty of Science, King Abdulaziz University, P.O. Box 80203, Jeddah 21589, Saudi Arabia

E-mail address: mkirane@univ-lr.fr 